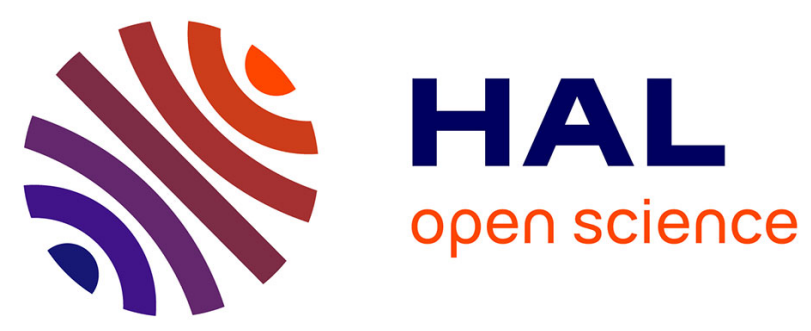

\title{
Augmented Lagrangian Method and Compressible Visco-Plastic Flows: Applications to Shallow Dense Avalanches
}

Didier Bresch, Enrique D. Fernandez - Nieto, Ioan R. Ionescu, Paul Vigneaux

\section{- To cite this version:}

Didier Bresch, Enrique D. Fernandez - Nieto, Ioan R. Ionescu, Paul Vigneaux. Augmented Lagrangian Method and Compressible Visco-Plastic Flows: Applications to Shallow Dense Avalanches. Fursikov, Andrei V., Galdi, Giovanni P., Pukhnachev, Vladislav V. New Directions in Mathematical Fluid Mechanics, Birkhäuser, pp.57-89, 2010, Advances in Mathematical Fluid Mechanics, 978-3-0346-01511. hal-00327369

\section{HAL Id: hal-00327369 \\ https://hal.science/hal-00327369}

Submitted on 20 Feb 2017

HAL is a multi-disciplinary open access archive for the deposit and dissemination of scientific research documents, whether they are published or not. The documents may come from teaching and research institutions in France or abroad, or from public or private research centers.
L'archive ouverte pluridisciplinaire HAL, est destinée au dépôt et à la diffusion de documents scientifiques de niveau recherche, publiés ou non, émanant des établissements d'enseignement et de recherche français ou étrangers, des laboratoires publics ou privés. 


\title{
Augmented Lagrangian Method and Compressible Visco-Plastic Flows: Applications to Shallow Dense Avalanches*
}

\author{
D. Breschł E.D. Fernández-Nieto \\ I.R. Ionescu $\stackrel{\S}{,}$ P. Vigneaux $\uparrow$ \\ Authors' manuscript - HAL version (February 2017) \\ Dedicated to the memory of Professor Alexander V. KAZHikov
}

\begin{abstract}
In this paper we propose a well-balanced finite volume / augmented Lagrangian method for compressible viscoplastic models focusing on a compressible Bingham type system with applications to dense avalanches. For the sake of completeness we also present a method showing that such system may be derived for a shallow flow of a rigid-viscoplastic incompressible fluid, namely for incompressible Bingham type fluid with free surface. When the fluid is relatively shallow and spread slowly, lubrication-style asymptotic approximations can be used to build reduced models for the spreading dynamics, see for instance [N.J. Balmforth et al., J. Fluid Mech. (2002)] . When the motion is a little bit quicker, shallow water theory for non Newtonian flows may be tried to handle with for instance assuming Navier type boundary condition at the bottom. We start from the variational inequality for incompressible Bingham fluid and derive a shallow water type system. In the case where Bingham number and viscosity are set to zero we obtain the classical Shallow Water or Saint-Venant equations obtained for instance in [J.F. Gerbeau \& B. Perthame, DCDS (2001)]. For numerical purposes, we focus on the one dimensional in space model: we study associated static solutions with sufficient condition that relates the slope of the bottom with the Bingham number and domain dimensions. We also propose a well-balanced finite volume / augmented Lagrangian method.
\end{abstract}

*This document is an improved version (some misprints corrected and changes of notations) of the article initially accepted in November, 2008 and published in Advances in Mathematical Fluid Mechanics. New Directions in Mathematical Fluid Mechanics, The Alexander V. Kazhikhov Memorial Volume (2010) pp. 57-89. Birkhäuser Basel / Springer.

†Laboratoire de Mathématiques, UMR 5127 CNRS, Univ. Savoie, 73376 Le Bourget du Lac (France). (Didier.Bresch@univ-savoie.fr)

${ }^{\ddagger}$ Dpto. Matemática Aplicada I, E.T.S. Arquitectura, U. Sevilla, Avda. Reina Mercedes n. 2, 41012 Sevilla (Spain) (edofer@us.es)

$\S$ Laboratoire P.M.T.M., Institut Galilée - Université Paris 13, Avenue Jean-Baptiste Clément 93430 Villetaneuse (France). (ioan.ionescu@lpmtm.univ-paris13.fr)

『Unité de Mathématiques Pures et Appliquées, ENS de Lyon, 46, allée d'Italie, 69364 Lyon Cedex 07 (France). (Paul.Vigneaux@math.cnrs.fr) 
It combines well-balanced finite volume schemes for the spacial discretization with the augmented Lagrangian method to treat the associated optimization problem. Finally, we present various numerical tests.

Keywords : Compressible flows, shallow water systems, viscoplastic flows, Bingham flows, avalanches, mixed finite volume/augmented Lagrangian, well-balanced scheme.

MSC2010: 35Q30 - 35Q86 - 49M29 - 65K15 - 76M12 - 76A05 - 74C10

Changes of notation from the original text:

- to harmonize with more standard nomenclatures, $\eta_{2} \rightarrow \tau_{y}, \kappa_{c} \rightarrow \tau_{c}, \eta_{1} \rightarrow \eta$

\section{Contents}

1 Introduction 2

2 Statement of the 3D problem 4

3 The plane slope case $\quad 6$

3.1 Momentum equation asymptotic . . . . . . . . . . . . . . . . . 9

4 One dimensional system and stationary solutions $\quad 10$

5 A well-balanced finite volume / augmented Lagrangian algorithm 12

5.1 Semi-discretization in time . . . . . . . . . . . . . . . 14

5.2 Rewriting the system: augmented Lagrangian . . . . . . . . . . . . . . . 14

5.3 Iterative algorithm for the saddle-point . . . . . . . . . . . . 16

5.4 Spatial discretization . . . . . . . . . . . . . . . . . . . . 17

5.4 .1 Definition of $\mathbf{b} \ldots \ldots \ldots \ldots$

5.4 .2 Approximation of $H^{n+1} \ldots \ldots \ldots . . \ldots 20$

5.4.3 Well-balanced properties of the proposed scheme . . . . . . . . 21

6 Numerical tests $\quad 22$

6.1 Test 1: convergence to a stationary solution . . . . . . . . . . . . 22

6.2 Test 2: transition between two stationary solutions . . . . . . . . . 23

6.3 Test 3: avalanche . . . . . . . . . . . . . . . . . . . . . 29

\section{Introduction}

Avalanches are natural phenomena that occur in mountainous regions such as Alps in France. During these last few years, we assist to real efforts devoted to the physical understanding of avalanche formation and motion in complex topography, see e.g. [2], [26], [3, 4, 24, 29]. This paper is an attempt to derive a compressible viscoplastic system from depth-averaged process for dense avalanches and to provide an accurate numerical 
scheme for such model. Our results concern two parts: A simple method to derive shallow water type model for an incompressible Bingham flow with free surface; A generalization to compressible flows of the Augmented Lagrangian method for incompressible Bingham viscoplastic flow initiated by R. GLOwInski, see [16]. Note that our numerical scheme may be used in other applications such as numerical modeling of projectile penetration into compressible rigid viscoplastic media, see for instance models in [13].

Remark that it is very difficult to postulate a constitutive relation for the stress tensor in terms of a deformation measure that correctly describes avalanches behaviour, see for instance [1]. This explain, for instance, why instead of prescribing a detailed constitutive relation, a Coulomb dry friction law for the basal friction and a Mohr-Coulomb yield criterion for the interior behavior have been used by several authors, see [26]. The information obtained in this way is sufficient to derive, more easily, dynamic equations that describe the spatio-temporal evolution of the height and the depth-averaged horizontal velocity component of the moving avalanche pile. In our paper, we propose to consider a shallow flow of a rigid viscoplastic incompressible fluid, namely a Bingham fluid [9]. More general constitutive relations may be studied such as those included in [13]. Remark also that depending on the basal boundary condition (slip boundary condition or non-slip boundary condition), various shallow water type equations may be obtained, see for instance [20] and [10] for models coming from incompressible Navier-Stokes equations and [18] for models coming from Bingham type equations with Dirichlet boundary condition at the bottom. Here, we consider boundary conditions in the spirit of [20], namely Navier boundary conditions at the bottom. This is dedicated to quicker flows replacing Dirichlet boundary conditions by a wall law boundary condition taking into account the boundary layer. Assuming Navier boundary condition, we start with the variational inequality for incompressible Bingham fluid and prove that a shallow water type system may be obtained using adequate test functions.

Several numerical simulations of avalanching flows in simple configuration are then proposed to compare our proposed scheme to previous ones. More precisely in our study, we propose a well-balanced finite volume / augmented Lagrangian method. It combines well-balanced finite volume schemes for the spacial discretization with the augmented Lagrangian method to treat the optimization problem. The key point in our result is that there exists a real interaction between the finite volume scheme and the augmented Lagrangian procedure. This gives a real well-balanced scheme that allows us to simulate initiation and run-out problems capturing interesting stationary solutions. Let us say that our numerical scheme will be soon tested in two-dimensional space interacting with C. ANCEY's group for experimental data. Readers interested by theoretical studies linked to compressible Bingham type models are referred to [7], [6], [5], [28] and more recently $[23]$.

This paper is organized as follows: in Section 2, we present the equations that define the 3D free-surface problem. In Section 3, we deduce the depth-averaged model from the variational inequality for incompressible Bingham fluid. In Section 4, we present the associated $1 \mathrm{D}$ system and sufficient conditions to identify stationary solutions of the model. The numerical scheme based on the combination of well-balanced finite volumes methods and the use of the augmented Lagrangian is shown in Section 5. Finally, in Section 6, we present three numerical tests. In the first one we study the convergence to 
a stationary solution when the initial profile of the free surface is a rectangular pulse. In this test we compare the results that we obtain with the proposed numerical scheme with non well-balanced numerical schemes. In the second numerical test we study the transition between two different stationary solutions corresponding to two different Bingham numbers. And in the third test we present the case of an avalanche over all the domain.

Let us finish this introduction by mentioning that this paper is dedicated to the Memory of Professor Alexander V. KAzHIKov, one of the most inventive applied mathematicians in compressible fluid mechanics.

\section{Statement of the 3D problem}

We consider here the evolution equations in the time interval $(0, T), T>0$ describing the flow of an inhomogeneous Bingham fluid in a domain $\mathcal{D}(t) \subset \mathbb{R}^{3}$ with a smooth boundary $\partial \mathcal{D}(t)$. In the following, the space and time coordinates as well as all mechanical fields are non dimensional. The notation $\boldsymbol{u}$ stands for the velocity field, $\boldsymbol{\sigma}$ denotes the Cauchy stress tensor field, $p=-\operatorname{trace}(\boldsymbol{\sigma}) / 3$ represents the pressure and $\boldsymbol{\sigma}^{\prime}=\boldsymbol{\sigma}+p I$ is the deviatoric part of the stress tensor. The momentum balance law in the Eulerian coordinates reads

$$
\rho\left(\operatorname{St} \frac{\partial \boldsymbol{u}}{\partial t}+(\boldsymbol{u} \cdot \nabla) \boldsymbol{u}\right)-\operatorname{div} \boldsymbol{\sigma}^{\prime}+\frac{1}{\mathrm{Fr}^{2}} \nabla p=\frac{1}{\mathrm{Fr}^{2}} \rho \boldsymbol{f} \quad \text { in } \mathcal{D}(t),
$$

where $\rho=\rho(t, x) \geq \underline{\rho}>0$ is the mass density distribution and $\boldsymbol{f}$ denotes the body forces. We have denoted by $\mathrm{St}=L_{c} /\left(V_{c} T_{c}\right), \mathrm{Fr}^{2}=V_{c}^{2} /\left(L_{c} f_{c}\right)$ the Strouhal and Froude numbers and we introduce $\mathrm{B}=\tau_{c} /\left(\rho_{c} V_{c}^{2}\right)$, where $\rho_{c}, V_{c}, L_{c}, \tau_{c}, T_{c}, f_{c}$ are the characteristic density, velocity, length, yield stress, time and force respectively. The characteristic pressure $p_{c}$ is assumed to be $p_{c}=\rho_{c} L_{c} f_{c}$, and the characteristic stress $\sigma_{c}=\rho_{c} V_{c}^{2}$. Since we deal with an incompressible fluid, we get

$$
\operatorname{div} \boldsymbol{u}=0 \quad \text { in } \mathcal{D}(t)
$$

The conservation of mass becomes

$$
\mathrm{St} \frac{\partial \rho}{\partial t}+\boldsymbol{u} \cdot \nabla \rho=0 \quad \text { in } \mathcal{D}(t)
$$

We notice from the above equation that, excepting some special cases, the flow of an incompressible fluid with inhomogeneous mass density is not stationary.

If we denote by $\boldsymbol{D}(\boldsymbol{u})=\left(\boldsymbol{\nabla} \boldsymbol{u}+\boldsymbol{\nabla}^{T} \boldsymbol{u}\right) / 2$ the rate of deformation tensor, the constitutive equation of the Bingham fluid can be written as follows:

$$
\begin{aligned}
\boldsymbol{\sigma}^{\prime} & =\frac{2}{\mathrm{Re}} \eta \boldsymbol{D}(\boldsymbol{u})+\mathrm{B} \tau_{y} \frac{\boldsymbol{D}(\boldsymbol{u})}{|\boldsymbol{D}(\boldsymbol{u})|} & & \text { if }|\boldsymbol{D}(\boldsymbol{u})| \neq 0, \\
\left|\boldsymbol{\sigma}^{\prime}\right| & \leq \mathrm{B} \tau_{y} & & \text { if }|\boldsymbol{D}(\boldsymbol{u})|=0,
\end{aligned}
$$

where $\eta \geq \eta_{0}>0$ is the non dimensional viscosity distribution depending on $\rho$ and $\tau_{y} \geq 0$ is a non-negative continuous function which stands for the non dimensional yield stress distribution in $\mathcal{D}(t)$. Here, $\operatorname{Re}=\rho_{c} V_{c} L_{c} / \eta_{c}$ is the Reynolds number and $\eta_{c}$ is a 
characteristic viscosity. Note that if $\tau_{c}$ is the characteristic yield stress then $\mathrm{B}=\mathrm{Bi} / \mathrm{Re}$, where $\mathrm{Bi}=\tau_{c} L_{c} /\left(\eta_{c} V_{c}\right)$ is the Bingham number. The type of behavior described by equations (4-5) can be observed in the case of some oils or sediments used in the process of oil drilling. The Bingham model, also denominated "Bingham solid" (see for instance [25]) was considered in order to describe the deformation of many solid bodies. Recently, the inhomogeneous (or density-dependent) Bingham fluid was chosen in landslides modeling $[15,12]$.

When considering a density-dependent model, the viscosity coefficient $\eta$ and the yield limit $\tau_{y}$ depend on the density $\rho$ through two constitutive functions, i.e.,

$$
\eta=\eta(\rho), \quad \tau_{y}=\tau_{y}(\rho) .
$$

In order to complete equations (1-6) with the boundary conditions, we assume that $\partial \mathcal{D}(t)$ is divided into two disjoint parts so that $\partial \mathcal{D}(t)=\Gamma_{b}(t) \cup \Gamma_{s}(t)$. On the boundary $\Gamma_{b}(t)$, which corresponds to the bottom part of the fluid, we consider a Navier condition with a friction coefficient $\alpha a$ and a no-penetration condition

$$
\boldsymbol{\sigma}_{t}=-\alpha a \boldsymbol{u}_{t}, \quad \boldsymbol{u} \cdot \boldsymbol{n}=0 \quad \text { on } \quad \Gamma_{b}(t),
$$

where $a$ is the non dimensional friction coefficient, $\alpha=a_{c} /\left(\rho_{c} V_{c}\right)$ and $a_{c}$ is the characteristic friction coefficient. Here, $\boldsymbol{n}$ stands for the outward unit normal on $\partial \mathcal{D}(t)$ and we have adopted the following notation for the tangential and normal decomposition of any velocity field $\boldsymbol{u}$ and any density of surface forces $\boldsymbol{\sigma} \boldsymbol{n}$ :

$$
\boldsymbol{u}=u_{n} \boldsymbol{n}+\boldsymbol{u}_{t} \text {, with } u_{n}=\boldsymbol{u} \cdot \boldsymbol{n}, \quad \boldsymbol{\sigma} \boldsymbol{n}=\sigma_{n} \boldsymbol{n}+\boldsymbol{\sigma}_{t} \text { with } \sigma_{n}=\boldsymbol{\sigma} \boldsymbol{n} \cdot \boldsymbol{n} .
$$

The (unknown) boundary $\Gamma_{s}(t)$ is a free surface, i.e. we assume a no-stress condition

$$
\boldsymbol{\sigma n}=0 \quad \text { on } \quad \Gamma_{s}(t),
$$

and the fact that the fluid region is advected by the flow, which can be expressed by

$$
\mathrm{St} \frac{\partial 1_{\mathcal{D}(t)}}{\partial t}+\boldsymbol{u} \cdot \nabla 1_{\mathcal{D}(t)}=0
$$

where $1_{\mathcal{D}(t)}$ is the characteristic function of the domain $\mathcal{D}(t)$.

Finally the initial conditions are given by

$$
\left.\boldsymbol{u}\right|_{t=0}=\boldsymbol{u}_{0},\left.\quad \rho\right|_{t=0}=\rho_{0}
$$

Setting

$$
\mathcal{V}(t)=\left\{\boldsymbol{\Phi} \in H^{1}(\mathcal{D}(t))^{3} / \operatorname{div} \boldsymbol{\Phi}=0 \text { in } \mathcal{D}(t), \quad \boldsymbol{\Phi} \cdot \boldsymbol{n}=0 \text { on } \Gamma_{b}(t)\right\},
$$

we give the variational formulation of (1), (2), (4), (5) and (7-8) for the velocity field (see 
[17]), namely

$$
\begin{aligned}
& \forall t \in(0, T), \quad \boldsymbol{u}(t, \cdot) \in \mathcal{V}(t), \quad \forall \boldsymbol{\Phi} \in \mathcal{V}(t), \\
& \int_{\mathcal{D}(t)} \rho\left(\mathrm{St} \frac{\partial \boldsymbol{u}}{\partial t}+(\boldsymbol{u} \cdot \nabla) \boldsymbol{u}\right) \cdot(\boldsymbol{\Phi}-\boldsymbol{u}) \\
& +\frac{1}{R e} \int_{\mathcal{D}(t)} 2 \eta(\rho) \boldsymbol{D}(\boldsymbol{u}):(\boldsymbol{D}(\boldsymbol{\Phi})-\boldsymbol{D}(\boldsymbol{u})) \\
& +B \int_{\mathcal{D}(t)} \tau_{y}(\rho)(|\boldsymbol{D}(\boldsymbol{\Phi})|-|\boldsymbol{D}(\boldsymbol{u})|) \\
& +\int_{\Gamma_{b}(t)} \alpha a \boldsymbol{u}_{t} \cdot\left(\boldsymbol{\Phi}_{t}-\boldsymbol{u}_{t}\right) \geq \frac{1}{F r^{2}} \int_{\mathcal{D}(t)} \rho \boldsymbol{f} \cdot(\boldsymbol{\Phi}-\boldsymbol{u}) .
\end{aligned}
$$

We can formulate the same problem in terms of velocity and pressure by using the space

$$
\mathcal{W}(t)=\left\{\boldsymbol{\Phi} \in H^{1}(\mathcal{D}(t))^{3} / \boldsymbol{\Phi} \cdot \boldsymbol{n}=0 \text { on } \Gamma_{b}(t)\right\}
$$

to deduce

$$
\left\{\begin{array}{l}
\forall t \in(0, T), \quad \boldsymbol{u}(t, \cdot) \in \mathcal{W}(t), p(t, \cdot) \in L^{2}(\mathcal{D}(t)), \quad \forall \boldsymbol{\Phi} \in \mathcal{W}(t), \forall q \in L^{2}(\mathcal{D}(t)), \\
\int_{\mathcal{D}(t)} \rho\left(\operatorname{St} \frac{\partial \boldsymbol{u}}{\partial t}+(\boldsymbol{u} \cdot \nabla) \boldsymbol{u}\right) \cdot(\boldsymbol{\Phi}-\boldsymbol{u})-\frac{1}{F r^{2}} \int_{\mathcal{D}(t)} p(\operatorname{div} \boldsymbol{\Phi}-\operatorname{div} \boldsymbol{u})+ \\
\frac{1}{R e} \int_{\mathcal{D}(t)} 2 \eta(\rho) \boldsymbol{D}(\boldsymbol{u}):(\boldsymbol{D}(\boldsymbol{\Phi})-\boldsymbol{D}(\boldsymbol{u}))+B \int_{\mathcal{D}(t)} \tau_{y}(\rho)(|\boldsymbol{D}(\boldsymbol{\Phi})|-|\boldsymbol{D}(\boldsymbol{u})|)+ \\
\int_{\Gamma_{b}(t)} \alpha a \boldsymbol{u}_{t} \cdot\left(\boldsymbol{\Phi}_{t}-\boldsymbol{u}_{t}\right) \geq \frac{1}{F r^{2}} \int_{\mathcal{D}(t)} \rho \boldsymbol{f} \cdot(\boldsymbol{\Phi}-\boldsymbol{u}), \\
\int_{\mathcal{D}(t)} q \operatorname{div} \boldsymbol{u}=0 .
\end{array}\right.
$$

Finally the problem of the flow of a inhomogeneous Bingham fluid becomes:

Find the velocity field $\boldsymbol{u}$ and the mass density field $\rho$ such that conditions (3), (6), (10) and (11) hold.

or in an equivalent form

Find the velocity field $\boldsymbol{u}$, the pressure $p$ and the mass density field $\rho$ such that conditions (3), (6), (10) and (12) hold.

As far as we know there does not exist any uniqueness result for this problem. Note recent mathematical studies in [7], [6], [5] and [28] dedicated to non-homogeneous incompressible Bingham flows and compressible Bingham flows in 1D space.

\section{The plane slope case}

We consider here the case of a plane slope. For this let $\Omega \subset \mathbb{R}^{2}$ be a fixed bounded domain and

$$
\mathcal{D}(t)=\{(x, z) ; x \in \Omega, 0<z<h(t, x)\},
$$


where $h(t, x)$ is the thickness of the fluid and $x=\left(x_{1}, x_{2}\right)$. We define by

$$
\Gamma_{s}(t)=\{(x, z) ; x \in \Omega, z=h(t, x)\}, \quad \Gamma_{b}(t)=\partial \mathcal{D}(t) \backslash \Gamma_{s}(t),
$$

the free and bottom surfaces, respectively. We denote by $\boldsymbol{v}=\left(v_{1}, v_{2}\right)$ the horizontal components of the velocity field and by $w$ the vertical one, i.e. $\boldsymbol{u}=(\boldsymbol{v}, w)$.

Penalization condition averaging. Let us remark that equation (9), for this choice of the flow geometry, reads

$$
\mathrm{St} \frac{\partial h}{\partial t}+\boldsymbol{v} \cdot \nabla_{x} h-w=0, \quad \text { for } z=h(t, x) .
$$

If we choose $q=q(x)$ dependent only on $x$ in (12) we get

$$
\begin{gathered}
0=\int_{\mathcal{D}(t)} q \operatorname{div} \boldsymbol{u}=\int_{\Omega} q(x)\left(\int_{0}^{h(t, x)} \operatorname{div}_{x} \boldsymbol{v}(t, x, z) d z+w(t, x, h(t, x))\right) d x= \\
\int_{\Omega} q(x)\left[\operatorname{div}_{x}\left(\int_{0}^{h(t, x)} \boldsymbol{v}(t, x, z) d z\right)-\boldsymbol{v}(t, x, h(t, x)) \cdot \nabla_{x} h(t, x)+w(t, x, h(t, x))\right] d x,
\end{gathered}
$$

and using the kinematic conditions (13) we get

$$
\int_{\Omega} q\left(\operatorname{St} \frac{\partial h}{\partial t}+\operatorname{div}_{x}(h \overline{\boldsymbol{v}})\right) d x=0, \quad \text { for all } q \in L^{2}(\Omega)
$$

where $\overline{\boldsymbol{v}}(t, x):=\frac{1}{h(t, x)} \int_{0}^{h(t, x)} \boldsymbol{v}(t, x, z) d z$ is the vertical mean value of the horizontal velocity.

Mass conservation equation averaging. Using the same technique as before one can deduce from the mass equation (3) that

$$
\int_{\Omega} q\left(\operatorname{St} \frac{\partial \bar{\rho} h}{\partial t}+\operatorname{div}_{x}(h \overline{\rho \boldsymbol{v}})\right) d x=0, \quad \text { for all } q \in L^{2}(\Omega),
$$

where $\bar{\rho}(t, x):=\frac{1}{h(t, x)} \int_{0}^{h(t, x)} \rho(t, x, z) d z$ is the vertical mean value of the mass density and $\overline{\rho \boldsymbol{v}}(t, x):=\frac{1}{h(t, x)} \int_{0}^{h(t, x)} \rho(t, x, z) \boldsymbol{v}(t, x, z) d z$ is the vertical mean value of the mass flux.

On the other hand the divergence free condition (2) reads

$$
w(t, x, z)=-\int_{0}^{z} \operatorname{div}_{x} \boldsymbol{v}(t, x, s) d s, \quad \text { for all }(x, z) \in \mathcal{D}(t) .
$$

Equation rescaling. In order to deduce an asymptotic model in the shallow flow approximation, we consider $\varepsilon \ll 1$ a small parameter representing the aspect ratio of the 
thickness. Following a standard scaling technique, we denote

$$
\begin{aligned}
& X:=x, \quad Z:=\frac{z}{H(t, x) \varepsilon}, \quad H:=\frac{h}{\varepsilon}, \quad \beta(t, X, Z):=\frac{\alpha a(t, x, z)}{\varepsilon}, \\
& \boldsymbol{V}(t, X, Z):=\boldsymbol{v}(t, x, z), \quad W(t, X, Z):=\frac{w(t, x, z)}{\varepsilon}, \quad P(t, X, Z):=p(t, x, z), \\
& \mathbf{F}_{X}(t, X, Z):=\boldsymbol{f}_{X}(t, x, z), \quad F_{Z}(t, X, Z):=\varepsilon f_{Z}(t, x, z),
\end{aligned}
$$

assuming $\boldsymbol{f}=\left(\boldsymbol{f}_{X}, f_{Z}\right)$ and a small slope angle leading to $\left|\boldsymbol{f}_{X}\right| \ll f_{Z}$. We denote by $\tilde{\mathcal{D}}$ the domain defined by $\Omega \times(0,1)$. In these new variables the equations (14-15) read

$$
\begin{gathered}
\operatorname{St} \frac{\partial H}{\partial t}+\operatorname{div}_{x}(H \overline{\boldsymbol{V}})=0, \\
\mathrm{St} \frac{\partial(\bar{\rho} H)}{\partial t}+\operatorname{div}_{x}(H \overline{\rho \boldsymbol{V}})=0,
\end{gathered}
$$

where

$$
\overline{\boldsymbol{V}}(t, x):=\int_{0}^{1} \boldsymbol{V}(t, x, Z) d Z
$$

and

$$
\overline{\rho \boldsymbol{V}}(t, x):=\int_{0}^{1} \varrho(t, x, Z) \boldsymbol{V}(t, x, Z) d Z
$$

are mean values on the thickness and we note $\varrho(t, x, Z)=\rho(t, x, z)$.

We write now each term of the variational inequality (12) in the scaled variables. For this we choose the same scaling for the test functions $\boldsymbol{\Phi}=(\boldsymbol{\Psi}, \varepsilon \theta)$. We decompose the left-hand side of (12) in five terms $I_{i}$ for $i=1, \ldots, 5$. They read

$$
\begin{gathered}
I_{1}=\varepsilon \int_{\tilde{\mathcal{D}}} H \varrho\left(\operatorname{St} \frac{\partial \boldsymbol{V}}{\partial t} \cdot(\boldsymbol{\Psi}-\boldsymbol{V})+\varepsilon^{2} \operatorname{St} \frac{\partial W}{\partial t}(\theta-W)\right) d X d Z \\
+\varepsilon \int_{\tilde{\mathcal{D}}} H \varrho\left(\left(\boldsymbol{V} \cdot \nabla_{x}\right) \boldsymbol{V}+\frac{1}{H} W \partial_{Z} \boldsymbol{V}\right) \cdot(\boldsymbol{\Psi}-\boldsymbol{V}) d X d Z \\
+\varepsilon^{3} \int_{\tilde{\mathcal{D}}} H \varrho\left(\boldsymbol{V} \cdot \nabla_{x} W+\frac{1}{H} W \partial_{Z} W\right)(\theta-W) d X d Z \\
I_{2}=\frac{\varepsilon}{\operatorname{Fr}^{2}} \int_{\tilde{\mathcal{D}}} H P\left(\operatorname{div}_{x} \boldsymbol{\Psi}+\frac{1}{H} \partial_{Z} \theta-\operatorname{div}_{x} \boldsymbol{V}-\frac{1}{H} \partial_{Z} W\right) d X d Z \\
I_{3}=\frac{\varepsilon}{\operatorname{Re}} \int_{\tilde{\mathcal{D}}} 2 \eta(\varrho)\left(H D(\boldsymbol{V}):(D(\boldsymbol{\Psi})-D(\boldsymbol{V}))+\frac{1}{H} \partial_{Z} W\left(\partial_{Z} \theta-\partial_{Z} W\right)\right) d X d Z \\
+\frac{\varepsilon}{\operatorname{Re}} \int_{\tilde{\mathcal{D}}}\left(\sum_{i=1}^{2} \eta(\varrho)\left(\varepsilon \partial_{x_{i}} W+\frac{1}{\varepsilon H} \partial_{Z} V_{i}\right)\left(\frac{1}{\varepsilon} \partial_{Z}\left(\boldsymbol{\Psi}_{i}-V_{i}\right)+\varepsilon H \partial_{x_{i}}(\theta-W)\right)\right) d X d Z
\end{gathered}
$$




$$
\begin{gathered}
I_{4}=-\varepsilon B \int_{\tilde{\mathcal{D}}} H \tau_{y}(\varrho)\left(\sqrt{|D(\boldsymbol{V})|^{2}+\left(\frac{1}{H} \partial_{Z} W\right)^{2}+\frac{1}{2} \sum_{i=1}^{2}\left(\varepsilon \partial_{x_{i}} W+\frac{1}{\varepsilon H} \partial_{Z} V_{i}\right)^{2}}\right. \\
-\sqrt{\left.|D(\boldsymbol{\Psi})|^{2}+\left(\frac{1}{H} \partial_{Z} \theta\right)^{2}+\frac{1}{2} \sum_{i=1}^{2}\left(\varepsilon \partial_{x_{i}} \theta+\frac{1}{\varepsilon H} \partial_{Z} \boldsymbol{\Psi}_{i}\right)^{2}\right)} d X d Z \\
I_{5}=\int_{\Omega} \varepsilon \beta \boldsymbol{V} \cdot(\boldsymbol{\Psi}-\boldsymbol{V}) d X .
\end{gathered}
$$

Concerning the right-hand side named $I_{6}$, we get

$$
I_{6}=\frac{\varepsilon}{F r^{2}} \int_{\tilde{\mathcal{D}}}\left(\varrho H \mathbf{F}_{X} \cdot(\boldsymbol{\Psi}-\boldsymbol{V})+\varrho H F_{Z}(\theta-W)\right) d X d Z .
$$

\subsection{Momentum equation asymptotic}

Let us assume that

$$
\mathrm{St}=\mathrm{Re}=\mathrm{B}=\mathrm{Fr}=\mathcal{O}(1), \quad \varepsilon \ll 1 .
$$

Dividing the variational inequality by $\varepsilon$, let us search for solutions, in the rescaled formulation, under the form

$$
\begin{aligned}
& \boldsymbol{V}=\boldsymbol{V}_{0}+\varepsilon \boldsymbol{V}_{1}+\cdots, \quad W=W_{0}+\varepsilon W_{1}+\cdots, \\
& P=P_{0}+\varepsilon P_{1}+\cdots, \quad \varrho=\rho_{0}+\varepsilon \rho_{1}+\cdots
\end{aligned}
$$

We denote $\boldsymbol{V}_{0}=\left(V_{0,1}, V_{0,2}\right)$. In what follows, we first focus on the terms of order $1 / \varepsilon^{2}$ and, then, we write the terms of order $\varepsilon^{0}$.

Terms of order $1 / \varepsilon^{2}$.

One gets

$$
\int_{\tilde{\mathcal{D}}} \frac{\eta\left(\rho_{0}\right)}{H} \partial_{Z} \boldsymbol{V}_{0} \cdot \partial_{Z}\left(\boldsymbol{\Psi}-\boldsymbol{V}_{0}\right)=0 .
$$

Assuming $\eta>c>0$ in $\tilde{\mathcal{D}}$, this gives using the boundary conditions

$$
\partial_{Z} V_{0,1}=\partial_{Z} V_{0,2}=0
$$

Terms of order $\varepsilon^{0}$.

Coming from (18)-(19), we get

$$
\begin{gathered}
\mathrm{St} \frac{\partial H}{\partial t}+\operatorname{div}\left(H \boldsymbol{V}_{0}\right)=0 \\
\operatorname{St} \frac{\partial\left(\overline{\rho_{0}} H\right)}{\partial t}+\operatorname{div}\left(H \overline{\rho_{0}} \boldsymbol{V}_{0}\right)=0 .
\end{gathered}
$$


Moreover, taking into account (25), the variational inequality becomes:

$$
\begin{aligned}
& \int_{\tilde{\mathcal{D}}} H \rho_{0}\left(\operatorname{St} \partial_{t} \boldsymbol{V}_{0}+\left(\boldsymbol{V}_{0} \cdot \nabla_{x}\right) \boldsymbol{V}_{0}\right) \cdot\left(\boldsymbol{\Psi}-\boldsymbol{V}_{0}\right) d X d Z+\int_{\Omega} \beta \boldsymbol{V}_{0} \cdot\left(\boldsymbol{\Psi}-\boldsymbol{V}_{0}\right) d X \\
& +\int_{\tilde{\mathcal{D}}}\left(\frac{2}{\operatorname{Re}} H \eta\left(\rho_{0}\right) D\left(\boldsymbol{V}_{0}\right): D\left(\boldsymbol{\Psi}-\boldsymbol{V}_{0}\right)+\frac{2}{\operatorname{Re}} \frac{1}{H} \eta\left(\rho_{0}\right) \partial_{Z} W_{0}\left(\partial_{Z} \theta-\partial_{Z} W_{0}\right)\right) d X d Z \\
& +\int_{\tilde{\mathcal{D}}} H \mathrm{~B} \tau_{y}\left(\rho_{0}\right)\left(\sqrt{|D(\boldsymbol{\Psi})|^{2}+\left(\frac{1}{H} \partial_{Z} \theta\right)^{2}}-\sqrt{\left|D\left(\boldsymbol{V}_{0}\right)\right|^{2}+\left(\frac{1}{H} \partial_{Z} W_{0}\right)^{2}}\right) d X d Z \\
& +\frac{1}{\operatorname{Fr}^{2}} \int_{\tilde{\mathcal{D}}} H P_{0}\left(\operatorname{div}_{x} \boldsymbol{\Psi}+\frac{1}{H} \partial_{Z} \theta\right) d X d Z-\frac{1}{\operatorname{Fr}^{2}} \int_{\tilde{\mathcal{D}}} H P_{0}\left(\operatorname{div}_{x} \boldsymbol{V}_{0}+\frac{1}{H} \partial_{Z} W_{0}\right) d X d Z \\
& \geq \frac{1}{\operatorname{Fr}^{2}} \int_{\tilde{\mathcal{D}}} H \rho_{0} \mathbf{F}_{X} \cdot\left(\boldsymbol{\Psi}-\boldsymbol{V}_{0}\right) d X d Z+\frac{1}{\operatorname{Fr}^{2}} \int_{\tilde{\mathcal{D}}} H \rho_{0} F_{Z}\left(\theta-W_{0}\right) d X d Z
\end{aligned}
$$

Using (25), $\operatorname{div}\left(\boldsymbol{V}_{0}, W_{0}\right)=0$ and the boundary conditions, we have $W_{0}=-Z H \operatorname{div}_{x} \boldsymbol{V}_{0}$. Moreover, in what follows we choose $\boldsymbol{\Psi}$ independent of $Z$. Finally, we choose the same relation for the test functions, that is $\theta=-Z H \operatorname{div}_{x} \Psi$. As $\tilde{\mathcal{D}}=\Omega \times(0,1)$, we can also integrate in $Z \in(0,1)$. We denote

$$
\begin{gathered}
\overline{\eta\left(\rho_{0}\right)}=\int_{0}^{1} \eta\left(\rho_{0}(Z)\right) d Z, \quad \overline{\tau_{y}\left(\rho_{0}\right)}=\int_{0}^{1} \tau_{y}\left(\rho_{0}(Z)\right) d Z, \\
\overline{\rho_{0} \mathbf{F}_{X}}=\int_{0}^{1} \rho_{0}(Z) \mathbf{F}_{X}(Z) d Z, \quad \overline{Z \rho_{0} F_{Z}}=\int_{0}^{1} Z \rho_{0}(Z) F_{Z}(Z) d Z .
\end{gathered}
$$

And we obtain

$$
\begin{aligned}
& \int_{\Omega} H \overline{\rho_{0}}\left(\operatorname{St} \partial_{t} \boldsymbol{V}_{0}+\left(\boldsymbol{V}_{0} \cdot \nabla_{x}\right) \boldsymbol{V}_{0}\right) \cdot\left(\boldsymbol{\Psi}-\boldsymbol{V}_{0}\right) d X+\int_{\Omega} \beta \boldsymbol{V}_{0} \cdot\left(\boldsymbol{\Psi}-\boldsymbol{V}_{0}\right) d X \\
& +\int_{\Omega} \frac{2}{\operatorname{Re}} H \overline{\eta\left(\rho_{0}\right)}\left(D\left(\boldsymbol{V}_{0}\right): D\left(\boldsymbol{\Psi}-\boldsymbol{V}_{0}\right)+\operatorname{div}_{x} \boldsymbol{V}_{0}\left(\operatorname{div}_{x} \boldsymbol{\Psi}-\operatorname{div}_{x} \boldsymbol{V}_{0}\right)\right) d X \\
& +\int_{\Omega} H \mathrm{~B} \overline{\tau_{y}\left(\rho_{0}\right)}\left(\sqrt{|D(\boldsymbol{\Psi})|^{2}+\left(\operatorname{div}_{x} \boldsymbol{\Psi}\right)^{2}}-\sqrt{\left|D\left(\boldsymbol{V}_{0}\right)\right|^{2}+\left(\operatorname{div}_{x} \boldsymbol{V}_{0}\right)^{2}}\right) d X \\
& \geq \frac{1}{\operatorname{Fr}^{2}} \int_{\Omega} H \overline{\rho_{0} \mathbf{F}_{X}} \cdot\left(\boldsymbol{\Psi}-\boldsymbol{V}_{0}\right) d X-\frac{1}{\operatorname{Fr}^{2}} \int_{\Omega} H^{2} \overline{Z \rho_{0} F_{Z}}\left(\operatorname{div}_{x} \boldsymbol{\Psi}-\operatorname{div}_{x} \boldsymbol{V}_{0}\right) d X
\end{aligned}
$$

Equation (29) with (26) and (27) gives a viscous shallow water formulation of Bingham type.

Remark 1. From (29), when $\tau_{y}=0$, we obtain an equality. In this case, we obtain a 2D viscous Shallow Water formulation, naturally associated to the Navier-Stokes equations, like in Gerbeau and Perthame [20].

\section{One dimensional system and stationary solutions}

In this section, we present the one dimensional in space system and its stationary solutions. In the next section, we present the numerical scheme to discretize this system and 
we study the well-balanced properties i.e. conditions allowing to preserve the stationary solution of the associated system.

From (26), (27) and (29), we obtain the one-dimensional in space model, if $H=$ $H(x, t), \overline{\rho_{0}}=\overline{\rho_{0}}(x, t)^{1}$ and $\boldsymbol{V}_{0}=\boldsymbol{V}_{0}(x, t)$, with $(x, t) \in[0, L] \times[0, T]$.

We consider the external forces

$$
F_{X}=-\sin \theta, \quad F_{Z}=-\varepsilon \cos \theta .
$$

Then one-dimensional in space model is defined by:

$$
\begin{gathered}
\operatorname{St} \frac{\partial H}{\partial t}+\frac{\partial\left(H \boldsymbol{V}_{0}\right)}{\partial x}=0 \\
\operatorname{St} \frac{\partial\left(\overline{\rho_{0}} H\right)}{\partial t}+\frac{\partial\left(H \overline{\rho_{0}} \boldsymbol{V}_{0}\right)}{\partial_{x}}=0 \\
\int_{0}^{L} H \overline{\rho_{0}}\left(\operatorname{St} \partial_{t} \boldsymbol{V}_{0}+\frac{1}{2} \partial_{x}\left(\boldsymbol{V}_{0}^{2}\right)\right)\left(\boldsymbol{\Psi}-\boldsymbol{V}_{0}\right) d x+\int_{0}^{L} \beta \boldsymbol{V}_{0}\left(\boldsymbol{\Psi}-\boldsymbol{V}_{0}\right) d x \\
+\int_{0}^{L} \frac{4}{\operatorname{Re}} H \overline{\eta\left(\rho_{0}\right)} \partial_{x}\left(\boldsymbol{V}_{0}\right) \partial_{x}\left(\boldsymbol{\Psi}-\boldsymbol{V}_{0}\right) d x+\int_{0}^{L} H \mathrm{~B} \overline{\tau_{y}\left(\rho_{0}\right)} \sqrt{2}\left(\left|\partial_{x} \boldsymbol{\Psi}\right|-\left|\partial_{x} \boldsymbol{V}_{0}\right|\right) d x \\
\geq \frac{-1}{\operatorname{Fr}^{2}} \int_{0}^{L} H \overline{\rho_{0}} \sin \theta\left(\boldsymbol{\Psi}-\boldsymbol{V}_{0}\right) d x+\frac{1}{\operatorname{Fr}^{2}} \int_{0}^{L} H^{2} \overline{\rho_{0}} \frac{\varepsilon \cos \theta}{2}\left(\partial_{x} \boldsymbol{\Psi}-\partial_{x} \boldsymbol{V}_{0}\right) d x .
\end{gathered}
$$

In what follows, we study sufficient conditions to ensure that a solution over an inclined slope is a stationary solution, with velocity being equal to zero.

By (32), we obtain that such a stationary solution must verify, for all $\mathbf{\Psi}$

$$
\int_{0}^{L} H \mathrm{~B} \overline{\tau_{y}\left(\rho_{0}\right)} \sqrt{2}\left|\partial_{x} \boldsymbol{\Psi}\right| \geq \frac{-1}{\mathrm{Fr}^{2}} \int_{0}^{L} H \overline{\rho_{0}} \sin \theta \boldsymbol{\Psi}+\frac{1}{\operatorname{Fr}^{2}} \int_{0}^{L} H^{2} \overline{\rho_{0}} \frac{\varepsilon \cos \theta}{2} \partial_{x} \Psi .
$$

Furthermore, we will focus on two types of solutions. The first one corresponds to material at rest, i.e. a stationary solution with velocity being equal zero and a horizontal free surface (See Figure 1). The property of horizontal free surface is defined by

$$
x \sin \theta+\varepsilon H \cos \theta=c s t,
$$

where $c$ st is the height of the free surface. Then, for this stationary solution, we obtain that the right hand side of (33) is equal to zero for all $\boldsymbol{\Psi}$. By the way, we deduce that the stationary solution corresponding to material at rest is a stationary solution of the model for all values of $\tau_{y}$ and $\theta$.

Secondly, we want to study sufficient conditions which ensure that a solution with a constant height over an inclined slope is stationary (See Figure 2). We define

$$
\mathcal{F}(x)=\frac{1}{\operatorname{Fr}^{2}} \int_{0}^{x} H \overline{\rho_{0}} \sin \theta d x+\frac{1}{\operatorname{Fr}^{2}} \frac{\overline{\rho_{0}}}{2} H^{2} \varepsilon \cos \theta .
$$

Then (33) can be rewritten as

$$
\int_{0}^{L} H \mathrm{~B} \overline{\tau_{y}\left(\rho_{0}\right)} \sqrt{2}\left|\partial_{x} \boldsymbol{\Psi}\right| d x \geq \int_{0}^{L} \mathcal{F}(x) \partial_{x} \boldsymbol{\Psi} d x .
$$

\footnotetext{
${ }^{1}$ From now on, we can actually already assume that $\rho_{0}$ does not depend on $Z$.
} 


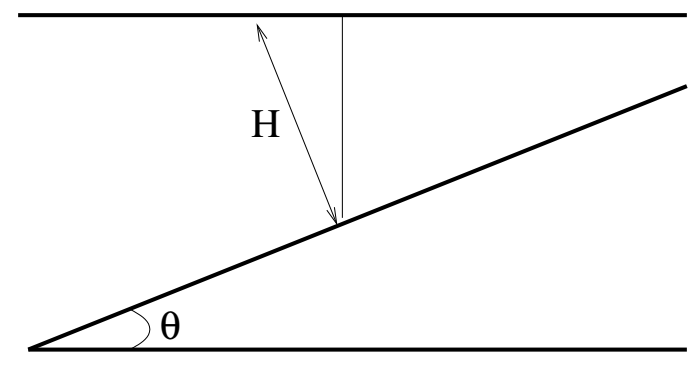

Figure 1: Stationary solution with horizontal free surface

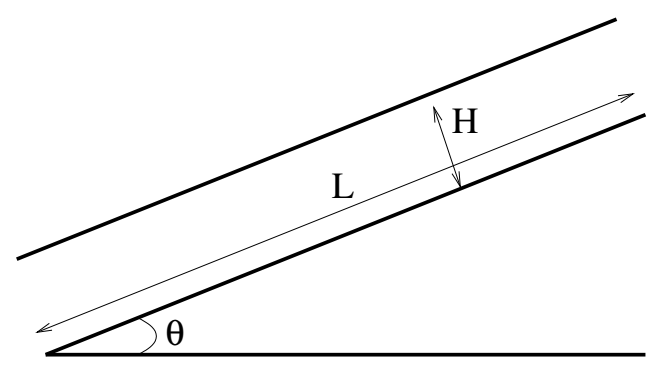

Figure 2: Stationary solution with constant height

And this inequality is satisfied if

$$
|\mathcal{F}(x)| \leq H \mathrm{~B} \overline{\tau_{y}\left(\rho_{0}\right)} \sqrt{2} .
$$

If $H$ is constant (as well as $\overline{\rho_{0}}$ ), then

$$
\mathcal{F}(x)=\frac{1}{\operatorname{Fr}^{2}} H \overline{\rho_{0}} \sin \theta x+\frac{1}{\operatorname{Fr}^{2}} \frac{\overline{\rho_{0}}}{2} H^{2} \varepsilon \cos \theta+c s t,
$$

where $c s t$ is a constant. Since we assume $H$ to be constant, we can then set

$$
\text { cst }=-\frac{1}{\mathrm{Fr}^{2}} H \overline{\rho_{0}} \sin \theta \frac{L}{2}-\frac{1}{\operatorname{Fr}^{2}} \frac{\overline{\rho_{0}}}{2} H^{2} \varepsilon \cos \theta .
$$

Finally, for this particular $\mathcal{F}$, we deduce that (34) and then (33) hold if

$$
\frac{1}{\operatorname{Fr}^{2}} \overline{\rho_{0}} \sin \theta \frac{L}{2} \leq B \overline{\tau_{y}\left(\rho_{0}\right)} \sqrt{2}
$$

Observe that if $\varepsilon=H / L$, previous condition can then be written as

$$
\frac{\overline{\rho_{0}}}{\operatorname{Fr}^{2}} H \sin \theta \leq 2 \sqrt{2} \varepsilon B \overline{\tau_{y}\left(\rho_{0}\right)} \text {. }
$$

\section{A well-balanced finite volume / augmented La- grangian algorithm}

In this section, we propose a numerical scheme to discretize the 1D model presented in the previous section. We consider the case with constant density, in such a way the 
model reduces to the equations (30) and (32) which contain the main difficulties.

First, we write the semi-discretization in time. Then, following [19], we observe that the problem (32) can be seen as an optimization problem and we use an augmented Lagrangian formulation to rewrite (32). This classically leads to the resolution of a saddle-point problem which involves an iterative method where are successively solved :

- a linear system associated to a problem on the speed (let us denote it as SU),

- and a minimization problem associated to the Lagrange multiplier (the solution of this problem is known explicitly).

The key-point of present paper consists in realizing that the global problem, which couples "indirectly" (30) and SU, implies that the numerical algorithms solving these two problems must also be coupled. Namely, their finite-volume spatial discretizations have terms in common. This is not obvious a priori, but if we look at the global problem, (30) and SU lead to an underlying Shallow Water system with source terms (linked to topography and Lagrangian terms). Consequently, if one wants the global scheme to preserve stationary solutions, philosophy inspired by so called "well-balanced" methods for Shallow Water system with source terms, must be used. Adopting these methods, SU is complemented with terms linked to the augmented Lagrangian, inducing a coupling which is - to our knowledge - not mentioned in previous works. In the following, we show that this method allows to perform simulations which preserve various stationary solutions (on the contrary to previous approaches for which we show that they are not well-balanced). Let us note that, actually, this method can be seen as a generalization to compressible flows of the augmented Lagrangian method for incompressible Bingham viscoplastic flow (applied, in the present paper, to Shallow Water type equations). In the context of non-integrated Navier-Stokes-Bingham, without Well-Balanced schemes, one can refer to [30].

Let us now precise further the underlying Shallow Water ideas which inspired our approach.

The accurate solution of hyperbolic systems with source terms requires numerical solvers with specific properties. Indeed, an upwind discretization of the source term, compatible with the one of the flux term, must be performed. Otherwise, a first order error in space, stemming from the numerical diffusion terms, takes place. This error, after time iterations, may yield large errors in wave amplitude and speed. RoE in [27] studies the relation between the choice of quadrature formulae to approximate the average of the source term and the property of preserving the stationary solutions.

BERMúdez and VÁZQUEZ-CÉNDón introduce in [8] some numerical solvers - with an upwind treatment of the source term for 1D Shallow Water equations (1D SWE) - which preserve water at rest. This work originated the so-called "well-balanced" solvers, in the sense that the discrete source terms balance the discrete flux terms when computed on some (or all) of the steady solutions of the continuous systems. Several sequels of this work for 1D SWE followed. See e.g. Greenberg-Leroux [21], LeVeque [22], Castro et al. [11]. 
This section is organized as follows. In Subsection 5.1, we present the semi-discretization in time of the model. In Subsection 5.2, we present the associated reformulation using an augmented Lagrangian method. We also discuss the link between the system obtained and the classical Shallow Water equations. In Subsection 5.3, we detail the iterative algorithm of the augmented Lagrangian. In Subsection 5.4, we introduce the spatial discretization. And finally, in Subsection 5.4.3, we study the well-balanced properties of the proposed numerical scheme.

\subsection{Semi-discretization in time}

We denote the variables with superscript $n$ to denote the approximation at time $t=t^{n}$ and with superscript $n+1$ for the time $t=t^{n}+\Delta t$.

Then, we consider the following semi-discretization in time of (30)-(32) :

$$
\begin{gathered}
\mathrm{St} \frac{H^{n+1}-H^{n}}{\Delta t}+\frac{\partial\left(H^{n} \boldsymbol{V}_{0}^{n}\right)}{\partial x}=0, \\
\int_{0}^{L} H^{n} \overline{\rho_{0}}\left(\operatorname{St} \frac{\boldsymbol{V}_{0}^{n+1}-\boldsymbol{V}_{0}^{n}}{\Delta t}\left(\boldsymbol{\Psi}-\boldsymbol{V}_{0}^{n+1}\right)+\frac{1}{2} \partial_{x}\left(\left(\boldsymbol{V}_{0}^{n}\right)^{2}\right)\left(\boldsymbol{\Psi}-\boldsymbol{V}_{0}^{n+1}\right)\right) d x \\
+\int_{0}^{L} \beta \boldsymbol{V}_{0}^{n+1}\left(\boldsymbol{\Psi}-\boldsymbol{V}_{0}^{n+1}\right) d x+\int_{0}^{L} \frac{4}{\operatorname{Re}} H^{n} \overline{\eta\left(\rho_{0}\right)} \partial_{x}\left(\boldsymbol{V}_{0}^{n+1}\right) \partial_{x}\left(\boldsymbol{\Psi}-\boldsymbol{V}_{0}^{n+1}\right) d x \\
+\int_{0}^{L} H^{n} \mathrm{~B} \overline{\tau_{y}\left(\rho_{0}\right)} \sqrt{2}\left(\left|\partial_{x}(\boldsymbol{\Psi})\right|-\left|\partial_{x}\left(\boldsymbol{V}_{0}^{n+1}\right)\right|\right) d x \\
\geq \frac{-1}{\operatorname{Fr}^{2}} \int_{0}^{L} H^{n} \overline{\rho_{0}} \sin \theta\left(\boldsymbol{\Psi}-\boldsymbol{V}_{0}^{n+1}\right)+\frac{1}{\operatorname{Fr}^{2}} \int_{0}^{L}\left(H^{n}\right)^{2} \overline{\rho_{0}} \frac{\varepsilon \cos \theta}{2}\left(\partial_{x} \boldsymbol{\Psi}-\partial_{x} \boldsymbol{V}_{0}^{n+1}\right) d x .
\end{gathered}
$$

\subsection{Rewriting the system: augmented Lagrangian}

We now follow ForTin \& GLOWINSKI [19] and rewrite equation (37) as an optimization problem : $\boldsymbol{V}_{0}^{n+1}$ is the solution of the minimization problem

$$
\mathcal{J}^{n}\left(\boldsymbol{V}_{0}^{n+1}\right)=\min _{\boldsymbol{V}} \mathcal{J}^{n}(\boldsymbol{V})
$$

where

$$
\mathcal{J}^{n}(\boldsymbol{V})=F^{n}(\mathcal{B}(\boldsymbol{V}))+G^{n}(\boldsymbol{V})
$$

with

$$
\begin{array}{lll}
\mathcal{B}: \mathcal{V} \rightarrow \mathcal{H} & F^{n}: \mathcal{H} \rightarrow \mathbb{R} & \text { with } \mathcal{V}=H_{0}^{1}([0, L]) \\
\mathcal{B}(\boldsymbol{V})=\partial_{x} \boldsymbol{V}, & F^{n}(\lambda)=\int_{0}^{L} H^{n} \mathrm{~B} \overline{\tau_{y}\left(\rho_{0}\right)} \sqrt{2}|\lambda| d x, & \text { and } \mathcal{H}=L^{2}([0, L]),
\end{array}
$$

and

$$
\begin{aligned}
G^{n}(\boldsymbol{V})= & \left.\int_{0}^{L} H^{n} \frac{\overline{\rho_{0}}}{(\mathrm{St}} \frac{\boldsymbol{V}^{2} / 2-\boldsymbol{V}_{0}^{n} \boldsymbol{V}}{\Delta t}+\frac{1}{2} \partial_{x}\left(\left(\boldsymbol{V}_{0}^{n}\right)^{2}\right) \boldsymbol{V}\right) d x \\
& +\int_{0}^{L} \beta \frac{\boldsymbol{V}^{2}}{2} d x+\int_{0}^{L} \frac{4}{\operatorname{Re}} H^{n} \overline{\eta\left(\rho_{0}\right)} \frac{1}{2}\left(\partial_{x} \boldsymbol{V}\right)^{2} d x \\
& +\frac{1}{\operatorname{Fr}^{2}} \int_{0}^{L} H^{n} \overline{\rho_{0}} \sin \theta \boldsymbol{V} d x-\frac{1}{\operatorname{Fr}^{2}} \int_{0}^{L}\left(H^{n}\right)^{2} \frac{\varepsilon \cos \theta}{2} \overline{\rho_{0}} \partial_{x} \boldsymbol{V} d x .
\end{aligned}
$$


Then, we define the Lagrangian by

$$
\begin{aligned}
& \mathcal{L}^{n}: \mathcal{V} \times \mathcal{H} \times \mathcal{H} \rightarrow \mathbb{R} \\
& \mathcal{L}^{n}(\boldsymbol{V}, q, \mu)=F^{n}(q)+G^{n}(\boldsymbol{V})+\int_{0}^{L} H^{n} \mu(\mathcal{B}(\boldsymbol{V})-q) d x,
\end{aligned}
$$

and the augmented Lagrangian, for a given value $r \in \mathbb{R}(r>0)$, is defined by

$$
\mathcal{L}_{r}^{n}(\boldsymbol{V}, q, \mu)=\mathcal{L}^{n}(\boldsymbol{V}, q, \mu)+\frac{r}{2} \int_{0}^{L} H^{n}(\mathcal{B}(\boldsymbol{V})-q)^{2} d x .
$$

Consequently the initial optimization problem consists now in characterizing the saddle point of $\mathcal{L}_{r}^{n}(\boldsymbol{V}, q, \mu)$. On the one hand, let us now begin by deriving with respect to $\boldsymbol{V}$ in (38). It reads

$$
\mathcal{M}(\boldsymbol{V}, q, \mu, \boldsymbol{\Psi})=0, \quad \forall \boldsymbol{\Psi}
$$

where

$$
\begin{aligned}
\mathcal{M}(\boldsymbol{V}, q, \mu, \boldsymbol{\Psi})= & \int_{0}^{L} H^{n} \overline{\rho_{0}}\left(\operatorname{St} \frac{\boldsymbol{V}-\boldsymbol{V}_{0}^{n}}{\Delta t} \boldsymbol{\Psi}+\frac{1}{2} \partial_{x}\left(\left(\boldsymbol{V}_{0}^{n}\right)^{2}\right) \boldsymbol{\Psi}\right) d x \\
& +\int_{0}^{L} \beta \boldsymbol{V} \boldsymbol{\Psi} d x+\int_{0}^{L} \frac{4}{\operatorname{Re}} H^{n} \overline{\eta\left(\rho_{0}\right)} \partial_{x}(\boldsymbol{V}) \partial_{x}(\boldsymbol{\Psi}) d x \\
& +\frac{1}{\operatorname{Fr}^{2}} \int_{0}^{L} H^{n} \overline{\rho_{0}} \sin \theta \boldsymbol{\Psi} d x \\
& -\frac{1}{\operatorname{Fr}^{2}} \int_{0}^{L}\left(H^{n}\right)^{2} \overline{\rho_{0}} \frac{\varepsilon \cos \theta}{2} \partial_{x} \boldsymbol{\Psi} d x+\int_{0}^{L} \mu H^{n} \mathcal{B}(\boldsymbol{\Psi}) d x \\
& +r \int_{0}^{L} H^{n}(\mathcal{B}(\boldsymbol{V})-q) \mathcal{B}(\boldsymbol{\Psi}) d x .
\end{aligned}
$$

On the other hand, as the problem is non differentiable with respect to $q$, we obtain the following variational inequality:

$$
\int_{0}^{L} H^{n} r q(p-q)+H^{n} \mathrm{~B} \overline{\tau_{y}\left(\rho_{0}\right)} \sqrt{2}(|p|-|q|)-H^{n}(\mu+r \mathcal{B}(\boldsymbol{V}))(p-q) d x \geq 0, \quad \forall p \in \mathcal{H}
$$

which can be rewritten as the following minimization problem: find $q \in \mathcal{H}$ solution of

$$
\min _{p \in \mathcal{H}}\left(\frac{H^{n} r}{2} p^{2}+H^{n} \mathrm{~B} \overline{\tau_{y}\left(\rho_{0}\right)} \sqrt{2}|p|-H^{n}(\mu+r \mathcal{B}(\boldsymbol{V})) p\right) .
$$

But this problem can be directly (and locally, for each $x \in[0, L]$ ) solved: the solution is

$$
q= \begin{cases}0 & \text { if }|\mu+r \mathcal{B}(\boldsymbol{V})|<\mathrm{B} \overline{\tau_{y}\left(\rho_{0}\right)} \sqrt{2}, \\ \frac{1}{r}\left((\mu+r \mathcal{B}(\boldsymbol{V}))-\mathrm{B} \overline{\tau_{y}\left(\rho_{0}\right)} \sqrt{2} \mathrm{SGN}(\mu+r \mathcal{B}(\boldsymbol{V}))\right) & \text { otherwise. }\end{cases}
$$


Moreover, from (39), we deduce that $\boldsymbol{V}$ verifies

$$
\begin{array}{r}
H^{n} \overline{\rho_{0}}\left(\operatorname{St} \frac{\boldsymbol{V}-\boldsymbol{V}_{0}^{n}}{\Delta t}+\frac{1}{2} \partial_{x}\left(\left(\boldsymbol{V}_{0}^{n}\right)^{2}\right)\right)+\partial_{x}\left(\frac{1}{\operatorname{Fr}^{2}}\left(H^{n}\right)^{2} \overline{\rho_{0}} \frac{\varepsilon \cos \theta}{2}\right)=-\beta \boldsymbol{V} \\
-\frac{1}{\operatorname{Fr}^{2}} H^{n} \overline{\rho_{0}} \sin \theta+\partial_{x}\left(H^{n}(\mu-r q)\right)+\partial_{x}\left(H^{n} \frac{\overline{4 \eta\left(\rho_{0}\right)}}{\operatorname{Re}} \partial_{x} \boldsymbol{V}\right)+\partial_{x}\left(H^{n} r \partial_{x} \boldsymbol{V}\right) .
\end{array}
$$

Finally, we observe that the new form of system (36)-(37) exhibits a coupling (through an iterative process described in the next subsection) of the following equations :

$$
\left\{\begin{array}{l}
\partial_{t} H+\partial_{x}(H \boldsymbol{V})=0 \\
H \overline{\rho_{0}} \operatorname{St} \partial_{t} \boldsymbol{V}+\frac{H \overline{\rho_{0}}}{2} \partial_{x}\left(\boldsymbol{V}^{2}\right)+\partial_{x}\left(\frac{1}{\operatorname{Fr}^{2}} H^{2} \overline{\rho_{0}} \frac{\varepsilon \cos \theta}{2}\right)=-\beta \boldsymbol{V} \\
-\frac{1}{\operatorname{Fr}^{2}} H \overline{\rho_{0}} \sin \theta+\partial_{x}(H(\mu-r q))+\partial_{x}\left(H \frac{\overline{4 \eta\left(\rho_{0}\right)}}{\operatorname{Re}} \partial_{x} \boldsymbol{V}\right)+\partial_{x}\left(H r \partial_{x} \boldsymbol{V}\right) .
\end{array}\right.
$$

Which are precisely the Shallow Water equations in formulation $(H, \boldsymbol{V})$, with viscosity, the source term defined by the topography and an extra source term linked to the augmented Lagrangian, namely :

$$
\partial_{x}(H(\mu-r q))
$$

where $\mu$ is the Lagrange multiplier and $q$ is the solution of the optimization problem (40), defined by (41).

\subsection{Iterative algorithm for the saddle-point}

We now present (still following [19]) the iterative algorithm used to compute the saddlepoint mentioned in the previous section.

We denote with superscripts $k$ and $k+1$ the variables involved in the iterative algorithm. Let us recall that, with superscript $n$ and $n+1$, we denote the approximations of the variables at time $t=t^{n}$ and $t=t^{n}+\Delta t$, respectively. The iterative algorithm is defined through the following steps:

\section{Step I.0 :}

We consider that we know $\boldsymbol{V}_{0}^{n}, H^{n}, \mu^{n}$ and $q^{n}$. Then, we impose for $k=0, \boldsymbol{V}^{k}=\boldsymbol{V}_{0}^{n}$, $\mu^{k}=\mu^{n}, q^{k}=q^{n}$.

\section{Step I.1 :}

Compute $q^{k+1}(x), \forall x \in[0, L]$ via :

$$
\begin{gathered}
d^{k+1}(x)=\left[\mu^{k}+r \mathcal{B}\left(\boldsymbol{V}^{k}\right)\right](x) \\
q^{k+1}(x)= \begin{cases}0 & \text { if }\left|d^{k+1}\right| \leq B \overline{\tau_{y}} \sqrt{2} \\
\frac{1}{r}\left(d^{k+1}-B \overline{\tau_{y}} \sqrt{2} \frac{d^{k+1}}{\left|d^{k+1}\right|}\right) & \text { if }\left|d^{k+1}\right|>B \overline{\tau_{y}} \sqrt{2}\end{cases}
\end{gathered}
$$




\section{Step I.2 :}

Compute $\boldsymbol{V}^{k+1}$ via :

$$
\begin{aligned}
& H^{n}\left[\overline{\rho_{0}} \operatorname{St} \frac{\boldsymbol{V}^{k+1}-\boldsymbol{V}_{0}^{n}}{\Delta t}+\partial_{x}\left(\frac{\overline{\rho_{0}}\left(\boldsymbol{V}_{0}^{n}\right)^{2}}{2}+\frac{1}{\operatorname{Fr}^{2}} H^{n} \overline{\rho_{0}} \varepsilon \cos \theta\right)\right]=-\beta \boldsymbol{V}^{k+1} \\
& -\frac{1}{\operatorname{Fr}^{2}} H^{n} \overline{\rho_{0}} \sin \theta+\partial_{x}\left(H^{n}\left(\mu^{k}-r q^{k+1}\right)\right)+\partial_{x}\left(H^{n}\left(\frac{4 \overline{\eta\left(\rho_{0}\right)}}{\operatorname{Re}}+r\right) \partial_{x} \boldsymbol{V}^{k+1}\right) .
\end{aligned}
$$

\section{Step I.3 :}

Update $\mu^{k+1}$ via :

$$
\mu^{k+1}=\mu^{k}+r\left(\mathcal{B}\left(\boldsymbol{V}^{k+1}\right)-q^{k+1}\right) .
$$

Loop Steps I.1-I.3 : $k \rightarrow k+1$

Until (e.g. with $\left.t o l=10^{-2}\right)$

$$
\frac{\left\|\mu^{k+1}-\mu^{k}\right\|}{\left\|\mu^{k}\right\|} \leq \text { tol. }
$$

\section{At convergence :}

We now have determined the value of $\boldsymbol{V}_{0}$ at time $t^{n+1}$, we just have to set :

$$
\boldsymbol{V}_{0}^{n+1}=\boldsymbol{V}^{k+1},
$$

and we also set $\mu^{n+1}=\mu^{k+1}, q^{n+1}=q^{k+1}$.

\subsection{Spatial discretization}

In this subsection, we describe the discretization in space of equations (36) and (46). That is, the spatial discretization of the following two equations:

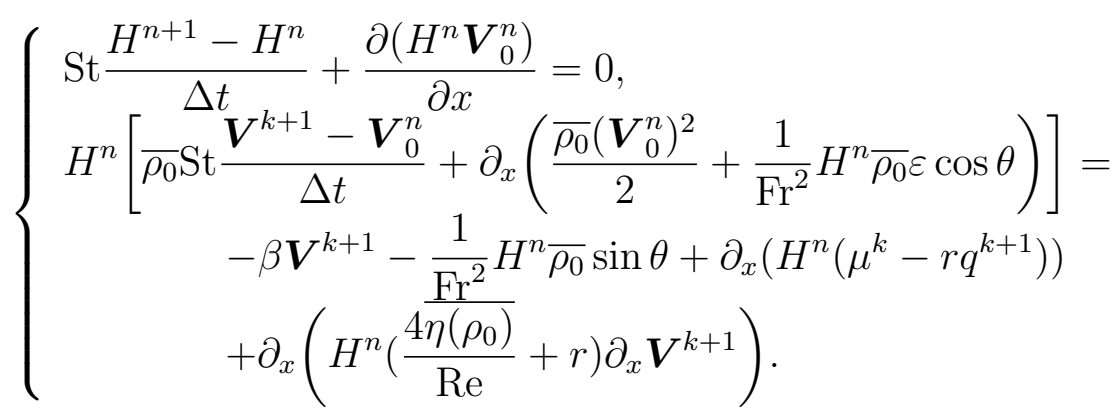

We observe that usually, to discretize Bingham system where two equations are involved, the discretization in space of both equations is uncoupled. See e.g. [14] for compressible Bingham system with variable density. Nevertheless, we propose a spatial discretization that contains a coupling between previous equations. As we mentioned previously, we want to obtain well-balanced numerical schemes. Basically, the difficulty to treat the spatial discretization in the present model comes from the extra source terms, which depends on $\mu$ and $q$.

In this section, we want to design a numerical scheme that preserves the following stationary solutions which can be encountered when using present model, namely : 
- Case $\tau_{y}=0$ : the model degenerates to Shallow Water equations (SWE) and the so called "water at rest" test case - where the velocity is equal to zero and the free surface is horizontal - is a classical solution. We thus want our scheme to degenerate to one of the well-balanced schemes for SWE ;

- Case $\tau_{y} \neq 0$ :

- Case "material at rest" : the solution to be captured for all $\tau_{y}>0$ is such that the velocity is equal to zero and the free surface is horizontal (cf. Figure 1) ;

- Case where the free surface has a constant height on an inclined slope ( $c f$. Figure 2) and the velocity is equal to zero is also a stationary solution under condition (35).

We note that the solution of the "water at rest" case and the one of the "material at rest" case are actually the same. Since our global model degenerates to the SWE when $\tau_{y}=0$, we want that, not only the solution in the case of a free surface with a constant height on an inclined slope, is rigorously captured by our scheme but also that the solution of the "material at rest" case (which seems to be rarely studied in the context of Bingham model). And it is worth noting that, on the one hand, designing a scheme that captures one or the other of these two solutions is quite easily achievable. On the other hand, a consistant scheme for the present model must preserve both solutions and the difficulty of its design lies behind this feature.

Let us go back for a while to the case $\tau_{y}=0$ - when the model reduces to SWE and follow the paper [11]. Namely, we remark that for Shallow Water model, the source term linked to the topography is only present in the momentum equations and does not appear in the mass conservation equation. Nevertheless, in well-balanced schemes, the topography term induces a contribution in the discretization of the mass conservation equation.

Taking into account the "Shallow Water structure" (mentioned in Section 5.2) of the present model, we borrow aforementioned idea by taking into account all the source terms of our momentum equation and plugging their contribution in the discretization of the mass conservation equation. In particular, and this is the key point of present approach, Lagrangian terms $\mu$ and $q$ will be in the discretization of the first equation of (50).

First, we rewrite the second equation of (50) as

$$
\begin{gathered}
H^{n} \overline{\rho_{0}} \mathrm{St} \frac{\boldsymbol{V}^{k+1}}{\Delta t}+\beta \boldsymbol{V}^{k+1}-\partial_{x}\left(H^{n}\left(\frac{4 \overline{\eta\left(\rho_{0}\right)}}{\mathrm{Re}}+r\right) \partial_{x} \boldsymbol{V}^{k+1}\right) \\
=-H^{n}\left[\partial_{x}\left(\frac{\overline{\rho_{0}}\left(\boldsymbol{V}_{0}^{n}\right)^{2}}{2}+\frac{1}{\operatorname{Fr}^{2}} H^{n} \overline{\rho_{0}} \varepsilon \cos \theta\right)-\overline{\rho_{0}} \mathrm{St} \frac{\boldsymbol{V}_{0}^{n}}{\Delta t}+\frac{1}{\operatorname{Fr}^{2}} \overline{\rho_{0}} \sin \theta\right] \\
+\partial_{x}\left(H^{n}\left(\mu^{k}-r q^{k+1}\right)\right) .
\end{gathered}
$$

For the right hand side of previous equation, we denote by $b_{i} \forall i$, a given approximation at the point $x_{i}$, i.e.

$$
b_{i} \approx\left\{-H^{n}\left[\partial_{x}\left(\frac{\overline{\rho_{0}}\left(\boldsymbol{V}_{0}^{n}\right)^{2}}{2}+\frac{1}{\operatorname{Fr}^{2}} H^{n} \overline{\rho_{0}} \varepsilon \cos \theta\right)\right.\right.
$$




$$
\left.\left.-\overline{\rho_{0}} \mathrm{St} \frac{\boldsymbol{V}_{0}^{n}}{\Delta t}+\frac{1}{\mathrm{Fr}^{2}} \overline{\rho_{0}} \sin \theta\right]+\partial_{x}\left(H^{n}\left(\mu^{k}-r q^{k+1}\right)\right)\right\}\left.\right|_{x=x_{i}} .
$$

In Subsection 5.4.1, we will introduce the approximation that we consider to define $b_{i}$.

Then, we define the vector $\mathbf{b}$ with all components, $\mathbf{b}:=\left(b_{i}\right)_{i}$. And we solve the linear system $A \mathbf{V}=\mathbf{b}$, where $A$ is the matrix of the system induced by (51). To define $A$ we consider a second order finite difference to approximate the left hand side of (51) and it reads

$$
\begin{gathered}
\left(\frac{H_{i}^{n} \overline{\rho_{0}} \mathrm{St}}{\Delta t}+\beta+\left(\frac{4 \overline{\eta\left(\rho_{0}\right)}}{R e}+r\right) \frac{H_{i+1 / 2}^{n}+H_{i-1 / 2}^{n}}{\Delta x^{2}}\right) \boldsymbol{V}_{i}^{k+1} \\
-\left(\frac{4 \overline{\eta\left(\rho_{0}\right)}}{R e}+r\right) \frac{H_{i+1 / 2}^{n}}{\Delta x^{2}} \boldsymbol{V}_{i+1}^{k+1}-\left(\frac{4 \overline{\eta\left(\rho_{0}\right)}}{R e}+r\right) \frac{H_{i-1 / 2}^{n}}{\Delta x^{2}} \boldsymbol{V}_{i-1}^{k+1},
\end{gathered}
$$

where $H_{i+1 / 2}^{n}=\left(H_{i}^{n}+H_{i+1}^{n}\right) / 2$.

\subsubsection{Definition of $\mathrm{b}$}

The definition of the right hand side in the linear system is fundamental in the design of the numerical scheme. For example, in relation with the stationary solutions of the system with velocity zero, $\mathbf{b}$ must be zero for all components.

We use a finite volume method to define b. In (52) we distinguish a component that is constant for the iterative algorithm in $k$ :

$$
-H^{n}\left[\partial_{x}\left(\frac{\overline{\rho_{0}}\left(\boldsymbol{V}_{0}^{n}\right)^{2}}{2}+\frac{1}{\operatorname{Fr}^{2}} H^{n} \overline{\rho_{0}} \varepsilon \cos \theta\right)-\overline{\rho_{0}} \mathrm{St} \frac{\boldsymbol{V}_{0}^{n}}{\Delta t}+\frac{1}{\mathrm{Fr}^{2}} \overline{\rho_{0}} \sin \theta\right] .
$$

And the first equation of (50) also contains the terms evaluated in $t=t^{n}$ :

$$
-\mathrm{St} \frac{H^{n}}{\Delta t}+\frac{\partial\left(H^{n} \boldsymbol{V}_{0}^{n}\right)}{\partial x}
$$

In the following, we denote by $F\left(H^{n}, \boldsymbol{V}^{n}\right)$ the flux function contained in equations (53)(54):

$$
F(W)=\left(\begin{array}{c}
H \boldsymbol{V} \\
\overline{\rho_{0}} \boldsymbol{V}^{2} / 2+H \overline{\rho_{0}} \varepsilon \cos \theta / \mathrm{Fr}^{2}
\end{array}\right), \quad \text { with } \quad W=\left(\begin{array}{c}
H \\
\boldsymbol{V}
\end{array}\right),
$$

and by $\phi$ a numerical flux function that approximates $F$. We begin by considering a family of numerical flux functions defined by (see [11]):

$$
\phi_{i+1 / 2}=\phi\left(W_{i}, W_{i+1}\right)=\frac{F\left(W_{i}\right)+F\left(W_{i+1}\right)}{2}-\frac{1}{2} D_{i+1 / 2}\left(W_{i+1}-W_{i}\right),
$$

where $D_{i+1 / 2}$ is a defined or semi-defined positive matrix. For example, the Lax-Friedrichs scheme corresponds to the definition $D_{i+1 / 2}:=\frac{\Delta x}{\Delta t} I$, where $I$ is the identity matrix. If we denote the Roe matrix by $\mathcal{J}_{i+1 / 2}$, then Roe method is obtained for $D_{i+1 / 2}=\left|\mathcal{J}_{i+1 / 2}\right|$, the absolute value of the Roe matrix associated to $F$. For the numerical results that we present in Section 6, we consider this scheme.

In the sequel, we denote by $\phi^{H}$ the first component of the numerical flux function and by $\phi^{V}$ the second one. The numerical flux function that we use is introduced in the following subsection and is defined in equation (59). 
By using the notation introduced previously we propose the following approximation of (52) to define $b_{i}$ :

$$
b_{i}=-H_{i}^{n}\left[\frac{\phi_{i+1 / 2}^{V}-\phi_{i-1 / 2}^{V}}{\Delta x}+\frac{1}{\mathrm{Fr}^{2}} \overline{\rho_{0}} \sin \theta-\mathrm{St} \frac{\overline{\rho_{0}} \boldsymbol{V}_{0, i}^{n}}{\Delta t}\right]+\frac{G_{i-1 / 2}^{V}+G_{i+1 / 2}^{V}}{2}
$$

with

$$
G_{i+1 / 2}^{V}=\frac{H_{i+1}^{n}\left(\mu_{i+1}^{k}-r q_{i+1}^{k+1}\right)-H_{i}^{n}\left(\mu_{i}^{k}-r q_{i}^{k+1}\right)}{\Delta x}
$$

\subsubsection{Approximation of $H^{n+1}$}

The first possible choice to define an approximation of $H^{n+1}$ is to use directly the first component of the flux function defined by (55). In this case we obtain

$$
\mathrm{St} H_{i}^{n+1}=\operatorname{St} H_{i}^{n}-\frac{\Delta t}{\Delta x}\left(\phi_{i+1 / 2}^{H}-\phi_{i-1 / 2}^{H}\right) .
$$

In the following, we denote the numerical scheme obtained in this case by (Non-WB $\mathbf{1}$ ), i.e. the first non well-balanced scheme. Since, actually, this scheme does not preserve the two types of stationary solutions. Namely, it is easy to prove that the scheme preserves the stationary solutions with constant height over an inclined plane. But, it does not preserve the stationary solutions with horizontal free surface.

Following [11], we can conclude that the source term which introduces the topography must be taken into account in the definition of $\phi^{H}$. If we denote

$$
G_{\text {topo }}=\left(\begin{array}{c}
0 \\
-\frac{1}{\operatorname{Fr}^{2}} \overline{\rho_{0}} \sin \theta
\end{array}\right)
$$

then $\phi^{H}$ is defined as the first component of

$$
\phi_{\text {topo }, i+1 / 2}=\frac{F\left(W_{i}\right)+F\left(W_{i+1}\right)}{2}-\frac{1}{2} D_{i+1 / 2}\left(W_{i+1}-W_{i}-\mathcal{J}_{i+1 / 2}^{-1} G_{\text {topo }}\right) .
$$

There are several techniques which are applied in the case where $\mathcal{J}_{i+1 / 2}$ is not inversible. For instance, one can define the eigenvalues of the generalized inverse matrix by zero if the corresponding eigenvalue to be inversed is null or smaller than a certain value of tolerance.

We can now introduce a second choice by defining $\phi^{H}$ as the first component of (58). This leads to another numerical scheme, which is denoted as (Non-WB 2), since it is not a well-balanced scheme. As a matter of fact, by introducing the technique proposed in [11] for SWE, we obtain that the scheme preserves the stationary solutions with horizontal free surface. But a constant height over an inclined plane is not a stationary solution of SWE, and we can prove that the obtained numerical scheme is not able to preserve these solutions.

Finally, we propose another discretization to define $\phi^{H}$ which leads to a scheme denoted as (WB-B). As we mentioned at the beginning of this section, the main difference 
between the scheme that we propose and previous ones is to treat Lagrangian variables $\mu$ and $q$ in the same manner as in the well-balanced schemes for SWE. Consequently, we propose to define $\phi^{H}$ by taking into account the term defined as a function of $\mu+r q$ as a source term. Namely, if the iterative algorithm ends for index $k_{e}$, we approximate previous term by $\mu^{k_{e}+1}+r q^{k_{e}+1}$. And we thus define $\phi^{H}$ as the first component of

$$
\begin{gathered}
\phi_{\mu, q, i+1 / 2}=\frac{F\left(W_{i}\right)+F\left(W_{i+1}\right)}{2}- \\
\frac{1}{2} D_{i+1 / 2}\left(W_{i+1}-W_{i}-\mathcal{J}_{i+1 / 2}^{-1}\left(G_{\text {topo }, i+1 / 2}+G_{\mu, q, i+1 / 2}\right)\right) .
\end{gathered}
$$

where

$$
G_{\mu, q, i+1 / 2}=\left(\begin{array}{c}
0 \\
H_{i+1}^{n}\left(\mu_{i+1}^{k_{e}+1}-r q_{i+1}^{k_{e}+1}\right)-H_{i}^{n}\left(\mu_{i}^{k_{e}+1}-r q_{i}^{k_{e}+1}\right)
\end{array}\right)
$$

\subsubsection{Well-balanced properties of the proposed scheme}

If we consider a domain of length $L$, we obtain the following result:

Theorem 5.1 If we consider the following initialization for $\mu$ and $q$ :

$$
\mu(x)=\frac{1}{\mathrm{Fr}^{2}} \overline{\rho_{0}} \sin \theta(x-L / 2)-\frac{1}{\mathrm{Fr}^{2}} \overline{\rho_{0}} \varepsilon \cos \theta(H(x)-H(L / 2)), \quad q(x)=0 \quad \forall x \in[0, L],
$$

then, the numerical scheme (WB-B) exactly preserves the stationary solution of material at rest, and also exactly preserves the stationary solution of constant height over an inclined plane verifying (35).

PROOF

To prove this result, it is enough to prove the following two items:

i) $\mathbf{b}=0$. In this case the solution of the linear system is $\boldsymbol{V}^{k+1}=0 \forall k$. So, if $\boldsymbol{V}^{n}=0$ we obtain that $\boldsymbol{V}^{n+1}=0$.

ii) $H_{i+1}^{n}-H_{i}^{n}-\left[\mathcal{J}_{i+1 / 2}^{-1}\left(G_{\text {topo }, i+1 / 2}+G_{\mu, q, i+1 / 2}\right)\right]_{1}=0 \forall i$. In this case, since $\boldsymbol{V}^{n+1}=0$, we obtain that $\phi_{i+1 / 2}^{H}$ defined by (59) is zero for all $i$, then by (57) we have that $H^{n+1}=H^{n}$.

Consequently, if $i$ ) and $i i$ ) are verified, the given stationary solution is exactly preserved. The verification of $i$ ) and $i i$ ) is an easy computation, so that, for the sake of brevity, we omit it.

Although we refer to two types of stationary solutions, in Section 6 we observe that the proposed scheme preserves other types of stationary solutions, even when there is a bump in the free surface and the Bingham number is relatively huge. In the Test 1 presented in Section 6, we compare the results obtained with (WB-B) and the non-well-balanced schemes (Non-WB 1) and (Non-WB 2). 


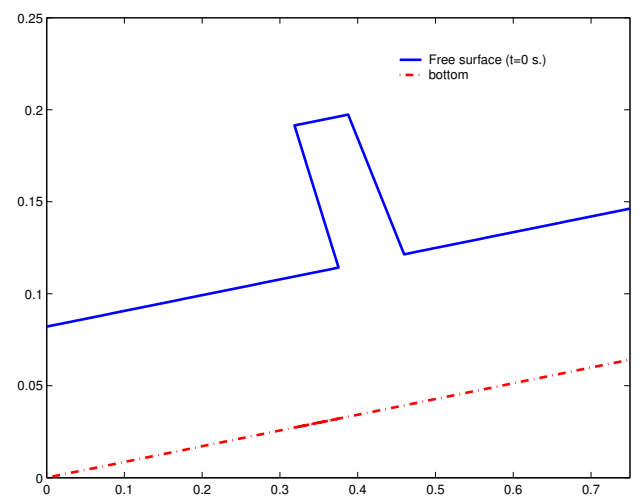

Figure 3: Test 1: Initial condition

\section{$6 \quad$ Numerical tests}

In this section, we present three numerical tests. In the first one, we study the convergence of a rectangular pulse towards the stationary solution, and the dependence of the stationary state on various Bingham numbers is explored. In the second numerical test, we present the transition between two stationary solutions when the rigid properties of the material change. In the third test, we present the case of an avalanche over the considered domain.

For the tests, we set the parameters $\mathrm{St}=\mathrm{B}=\mathrm{Re}=1, \varepsilon=1, \eta=1$ and $\mathrm{Fr}=0.3193$. Moreover, CFL condition is equal to $0.8, r=\Delta x \tau_{y} / \eta$ and $\Delta x=0.01$. We consider different values of $\tau_{y}$ in the following numerical tests. For the boundary conditions we impose the velocity to be zero.

\subsection{Test 1: convergence to a stationary solution}

In this subsection, we present a test where the free surface of the initial condition is a rectangular pulse and the initial velocity is equal to zero in all the domain. Furthermore, the bottom is supposed to be an inclined plane with an angle of 5 degrees ( $c f$. Figure 3 ). We study the final stationary profile of the material surface and the dependence of the shape with respect to different Bingham numbers.

The domain considered is $[0,1]$. The height of the material is defined by:

$$
H(x, 0)= \begin{cases}0.2 & \text { if } x \in[0.5,0.6] \\ 0.1 & \text { otherwise }\end{cases}
$$

We study the evolution towards a stationary solution for different values of the Bingham number. Namely, we consider $\tau_{y}=10,2,0.1$ and 0.01 . The goal is to study the rigid properties of the material with respect to the Bingham number. When the Bingham number is nearly zero, the material is more similar to a fluid like the water. In fact, it can be remembered that when $\tau_{y}=0$ the proposed model reduces to Shallow Water equations with viscous terms.

First, in Figure 4, we present the evolution at four different times of the material surface for $\tau_{y}=10$. Figure $4(\mathrm{~d})$ corresponds to $t=5 \mathrm{~s}$., where the solution is stationary. 
We observe that the stationary solution presents a bump, i.e. the material is sufficiently rigid to support the gradient of pressure produced by the gradient of the surface.

In Figure 5, we compare the numerical result obtained for $\tau_{y}=10$ with the proposed well-balanced scheme (continuous black line) and the results obtained without a wellbalanced treatment for the discretization of $H$. Namely, we compare with the schemes (Non-WB 1) (red doted lines of Figure 5) and (Non-WB 2) (blue dashed lines of Figure 5) presented in Section 5. Both schemes are not able to preserve the stationary solution with a bump in the free surface.

In Figure 6, we present the evolution of the surface for the four different values of $\tau_{y}$ $\left(\tau_{y}=10,2,0.1\right.$ and 0.01$)$. In Figure 7 , we present the stationary surfaces obtained with these values of $\tau_{y}$.

If we compare figures $7(\mathrm{a})$ with $7(\mathrm{~b})$, corresponding to $\tau_{y}=10$ and $\tau_{y}=2$, respectively, we observe that in both cases the stationary solution present a bump in the surface. Nevertheless the bump is smaller when $\tau_{y}$ decreases.

Figures $7(\mathrm{c})$ and $7(\mathrm{~d})$ correspond to $\tau_{y}=0.1$ and $\tau_{y}=0.01$, respectively. We observe that when $\tau_{y}$ converges to zero, the stationary solution converges to the stationary solution of water at rest over an inclined plane. Namely, they converges to the stationary solution that we obtain for this test with Shallow Water equations, i.e. the model obtained for $\tau_{y}=0$.

In Figure 8, we present the velocity at different times obtained with the four different values of $\tau_{y}$. We observe that the smaller values of velocities are obtained when the Bingham number is greater. Namely, for $t=0.5 \mathrm{~s}$. (Figure $8(\mathrm{a})$ ) we observe that the velocity is nearly zero for $\tau_{y}=10$. And for $t=1 \mathrm{~s}$. (Figure 8(a)) the solution corresponding to $\tau_{y}=10$ is vanishing, while for the other values of $\tau_{y}$ the velocity is, by comparison, far from zero. For $t=5 \mathrm{~s}$. (Figure 8(c)) the solution for $\tau_{y}=2$ exhibits a vanishing motion. We observe that for $t=20 \mathrm{~s}$. (Figure $8(\mathrm{~d})$ ) the velocities corresponding to $\tau_{y}=0.1$ and $\tau_{y}=0.01$ tend much more slowly to zero than the ones for $\tau_{y}=2$ and $\tau_{y}=10$.

Finally, in Figure 9 we present two comparisons. In Figure 9(a), we compare the four stationary solutions corresponding to the fours considered values of $\tau_{y}$. And, in Figure 9(b), we present the values of $\mu$ obtained at the final time, when the solution is stationary, for these values of $\tau_{y}$. We can easily identify that $\mu$ follows the same type of profile as the surface. Actually, as we mentioned previously, $\mu$ is a term whose role is to introduce an equilibrium in the pressure. If we observe for instance the case $\tau_{y}=10$, the material surface exhibits a gradient, which in the model of Shallow Water corresponds to a pressure gradient. This pressure gradient for stationary solutions is compensated, in the proposed model, by $\mu$. That is why, in the case of a stationary solution, the profile of the surface is of the same type as the one of $\mu$. Equivalently we observe that for $\tau_{y}=0.01$ where the material surface is nearly flat, the gradient of pressure is nearly zero, then the pressure gradient which has to be compensated is small, hence the profile of $\mu$ is close to zero.

\subsection{Test 2: transition between two stationary solutions}

The objective of this test is to observe the behaviour of the material when its rigid properties change. For instance, if we think about the snow, many phenomena can 


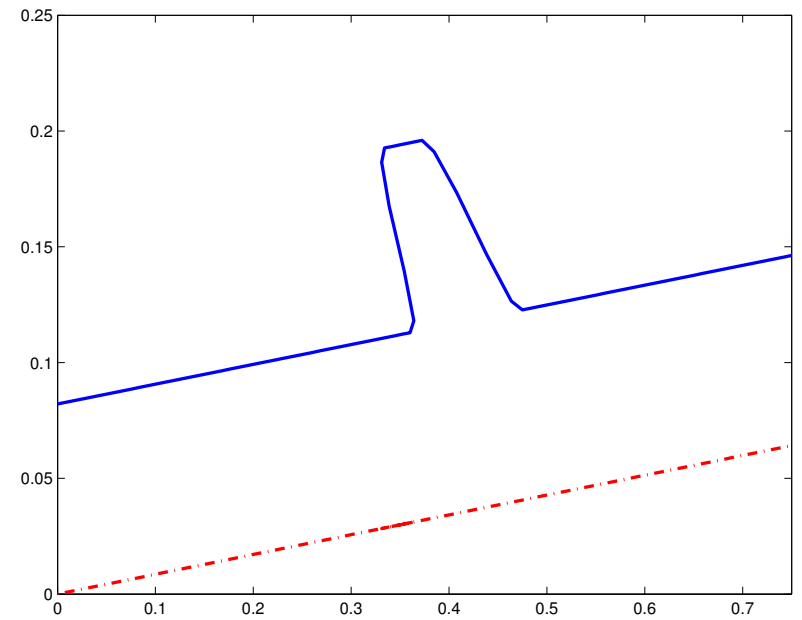

(a) $\mathrm{t}=0.01 \mathrm{~s}$.

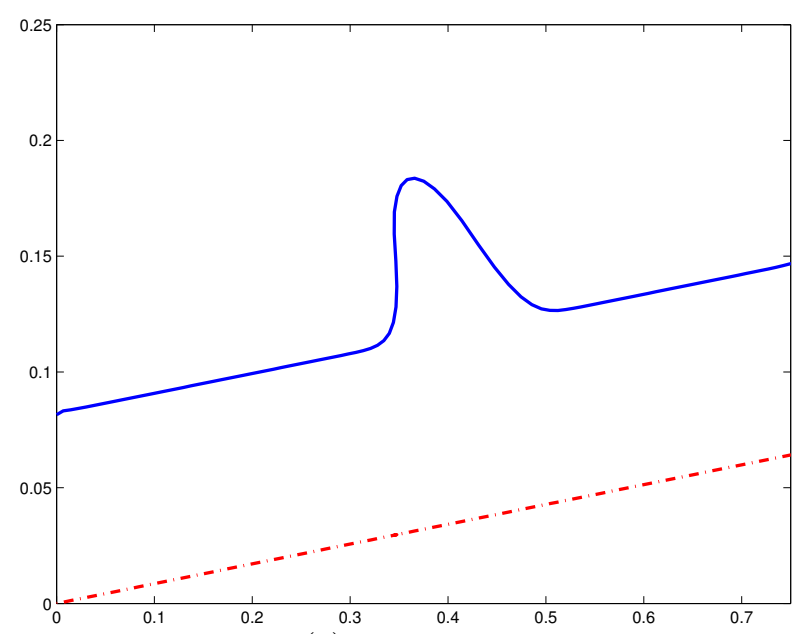

(c) $\mathrm{t}=0.1 \mathrm{~s}$.

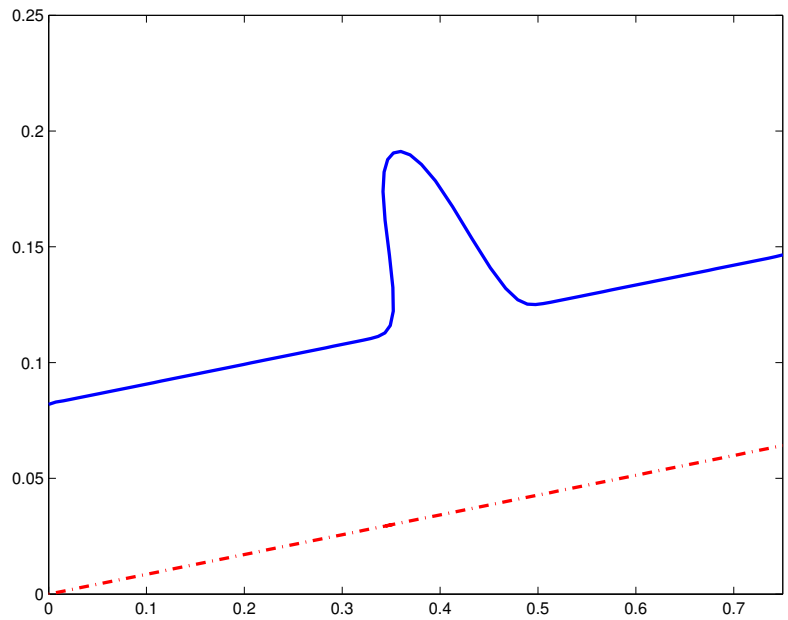

(b) $\mathrm{t}=0.05 \mathrm{~s}$.

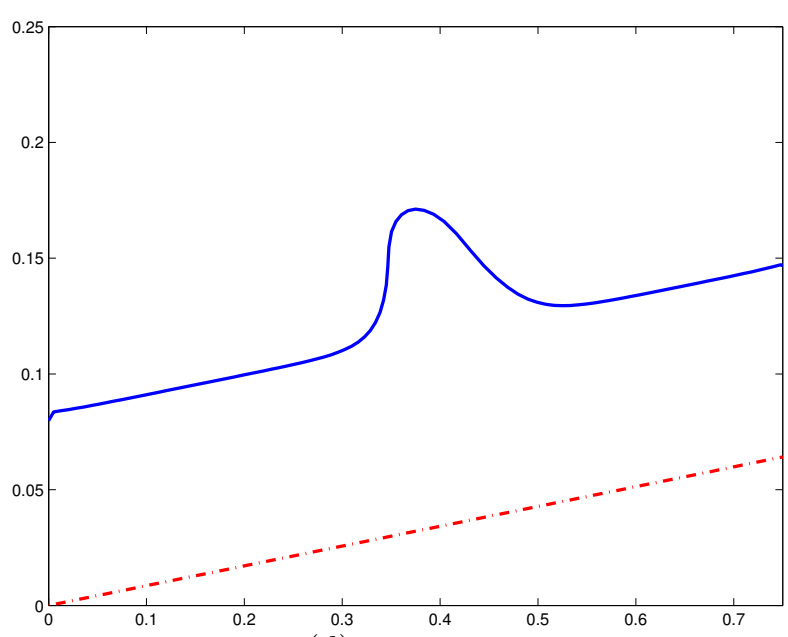

(d) $\mathrm{t}=5 \mathrm{~s}$.

Figure 4: Test 1: Evolution of the material surface $\tau_{y}=10$. 


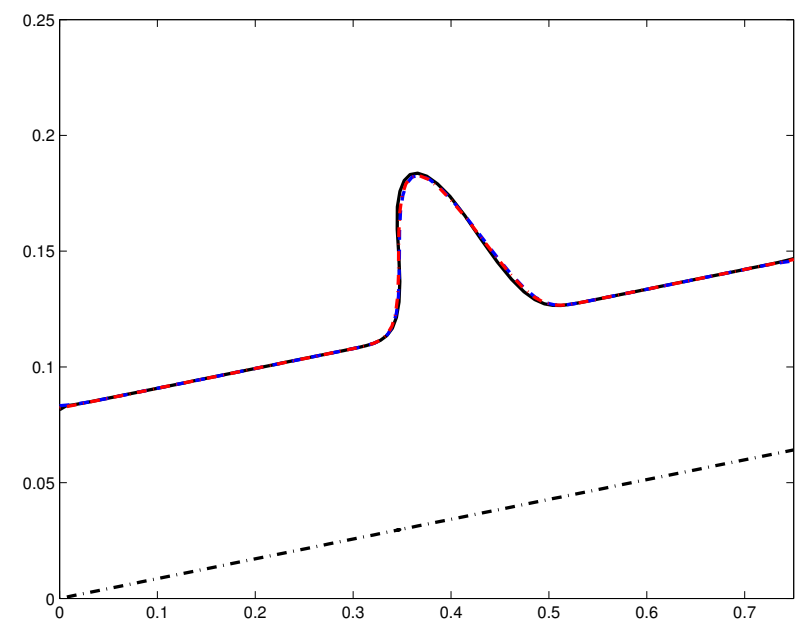

(a) $\mathrm{t}=0.1 \mathrm{~s}$.

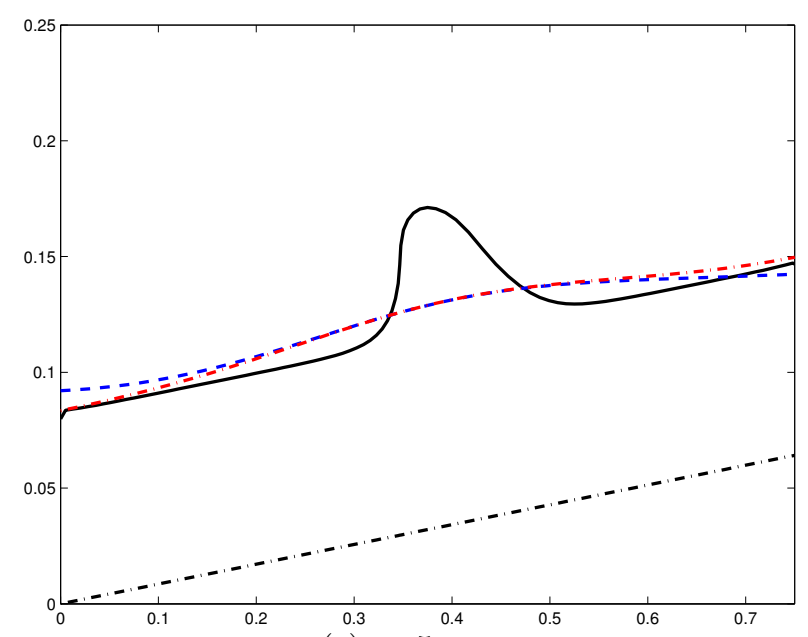

(c) $\mathrm{t}=5 \mathrm{~s}$.

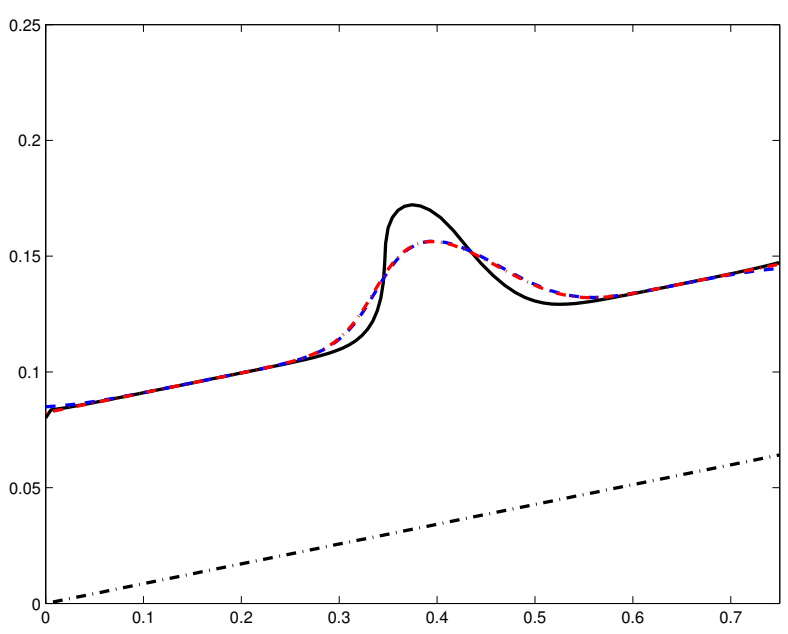

(b) $\mathrm{t}=0.5 \mathrm{~s}$.

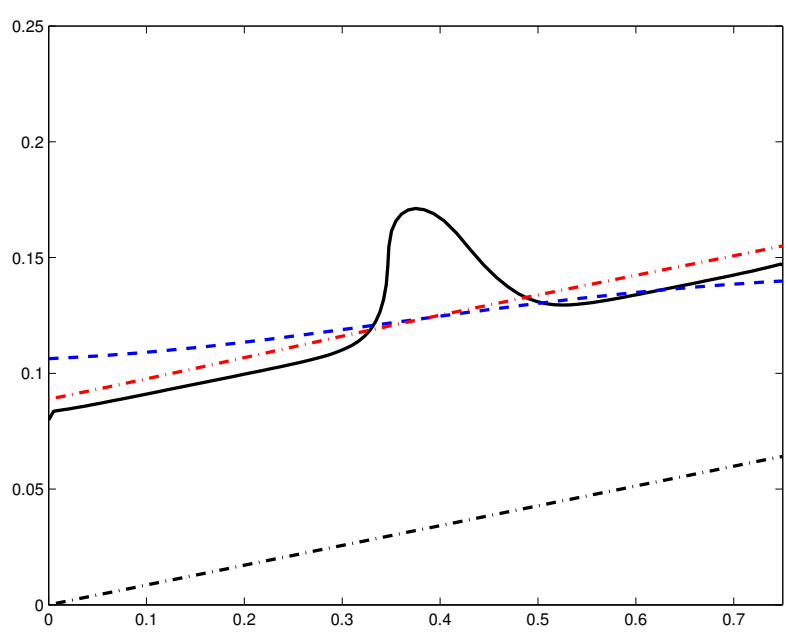

(d) $\mathrm{t}=20 \mathrm{~s}$.

Figure 5: Test 1: Evolution of the material surface $\tau_{y}=10$. Comparison between the numerical result of the well-balanced scheme (continuous black line) and the non wellbalanced schemes (Non-WB 1) (discontinuous red line) and (Non-WB 2) (dashed blue line) 


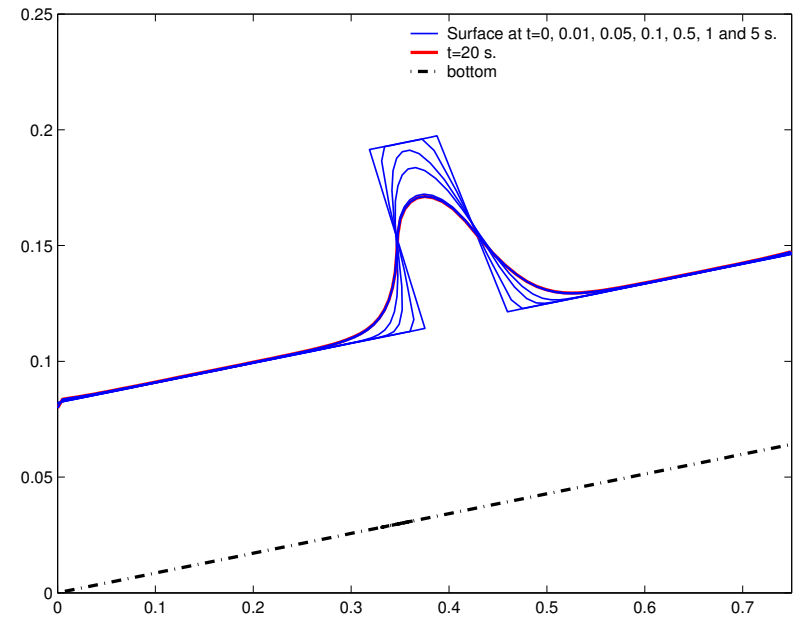

(a) $\tau_{y}=10$.

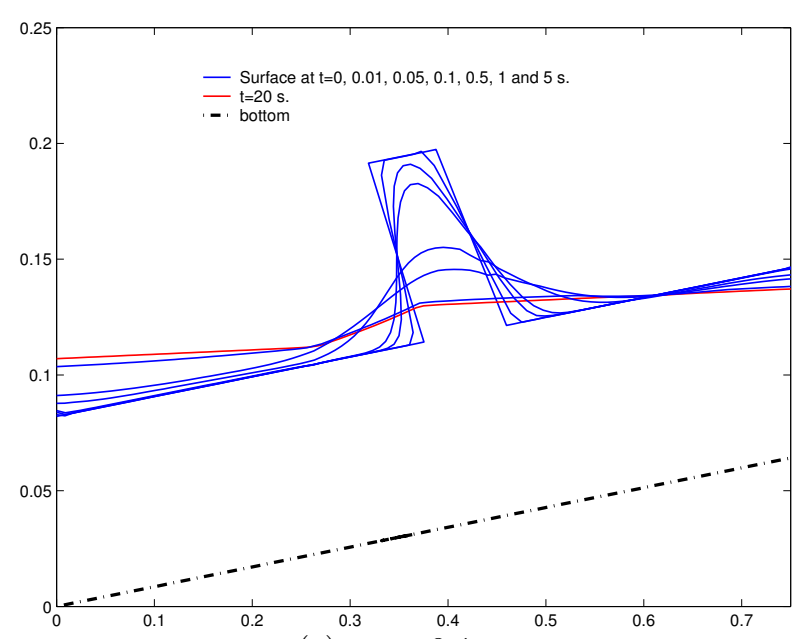

(c) $\tau_{y}=0.1$.

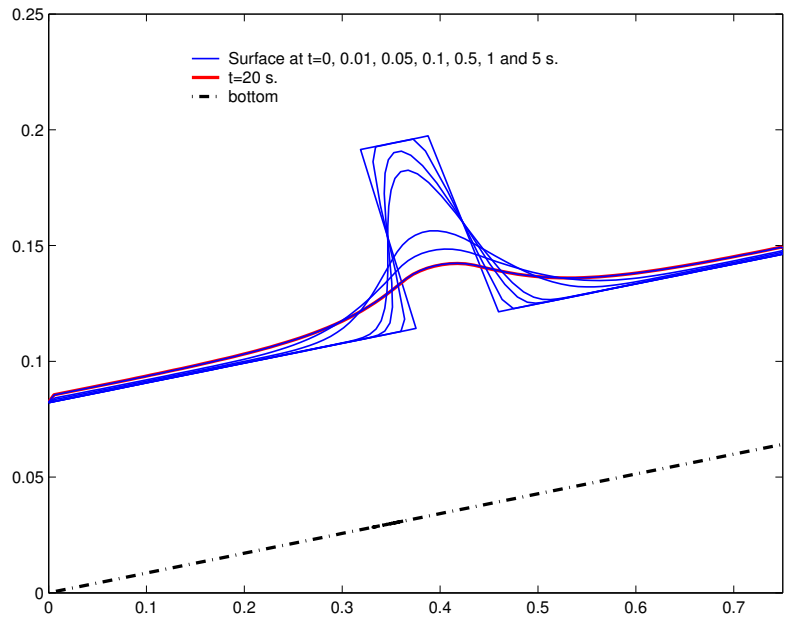

(b) $\tau_{y}=2$.

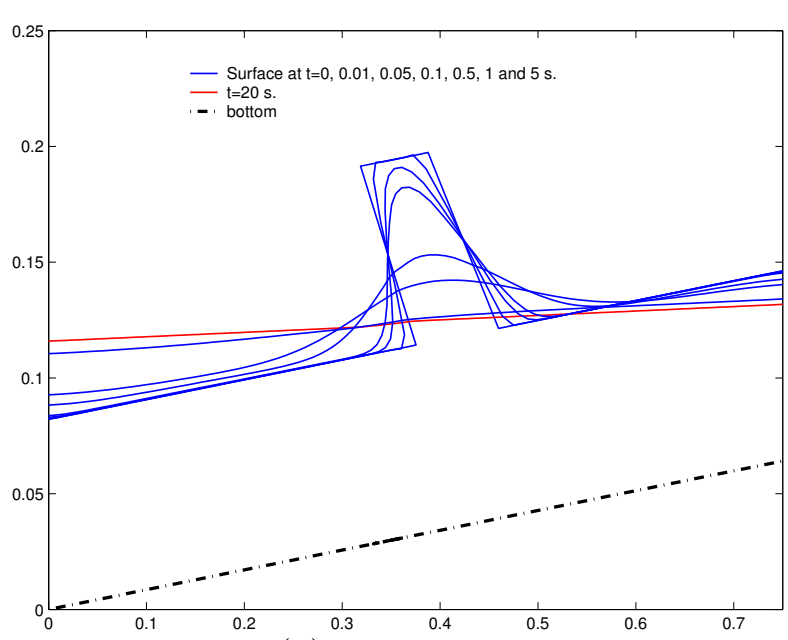

(d) $\tau_{y}=0.01$.

Figure 6: Test 1: Evolution of the free surface for $\tau_{y}=10,2,0.1$ and 0.01 


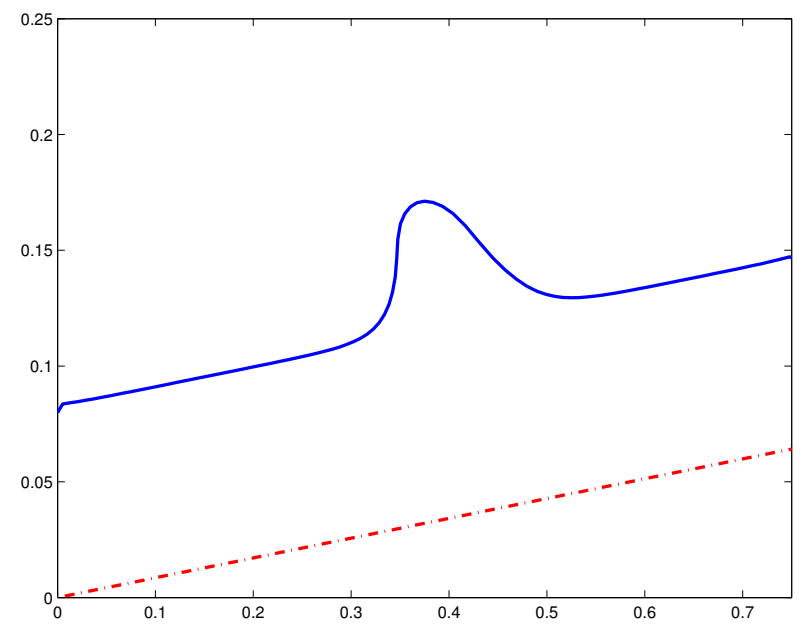

(a) $\tau_{y}=10$.

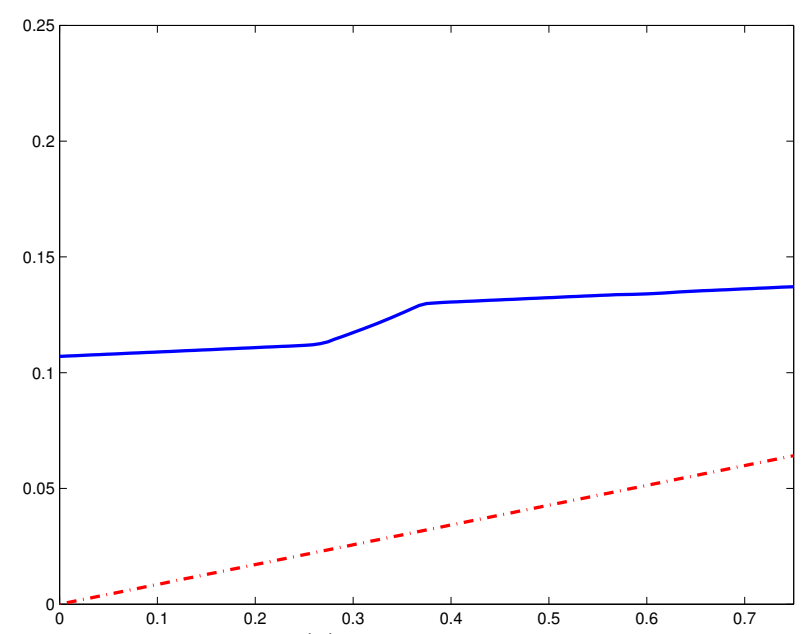

(c) $\tau_{y}=0.1$.

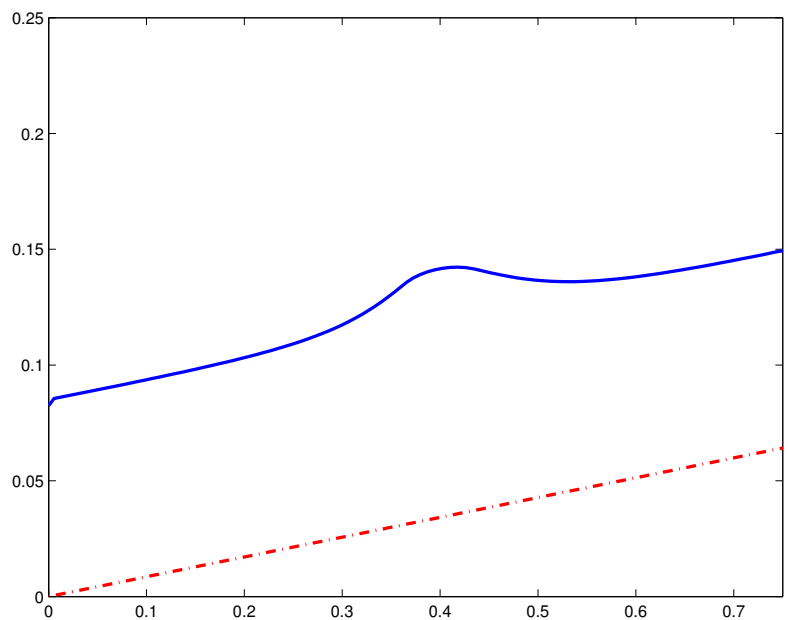

(b) $\tau_{y}=2$.

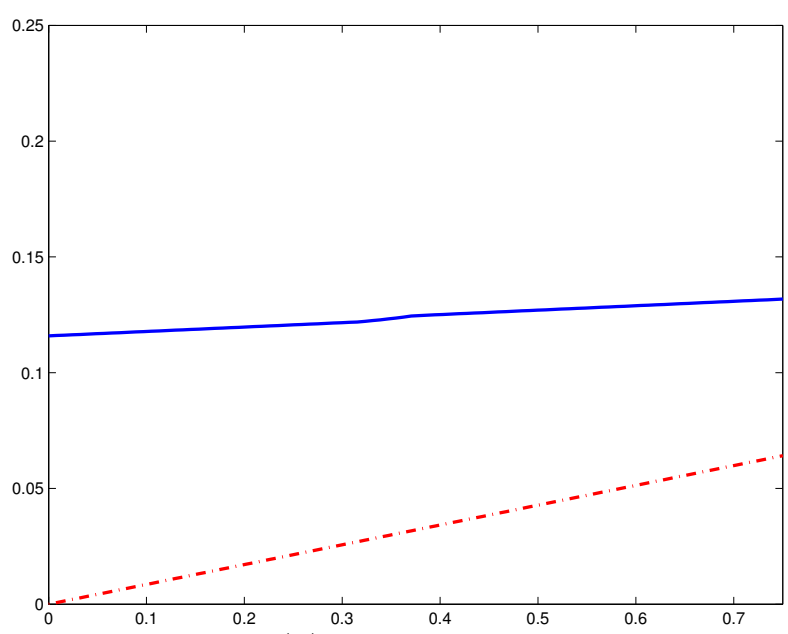

(d) $\tau_{y}=0.01$.

Figure 7: Test 1: Material surface of the stationary solutions obtained with $\tau_{y}=10,2$, 0.1 and 0.01 


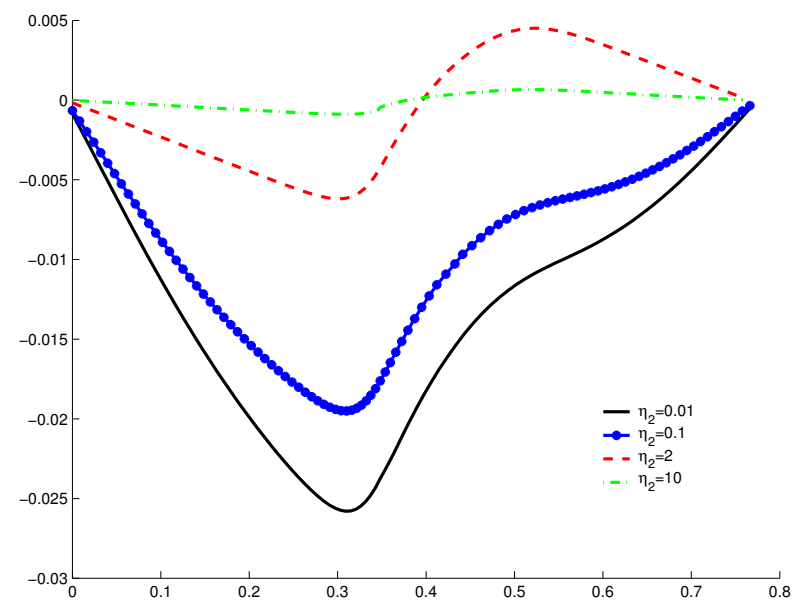

(a) $t=0.5$.

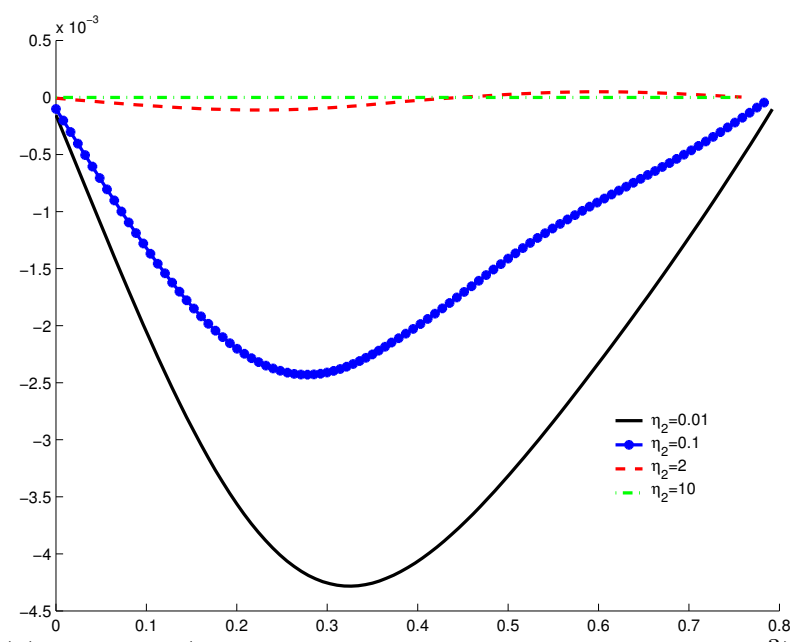

(c) $t=5 \mathrm{~s}$. (Range of the $y$-axis multiplied by $10^{-3}$ )

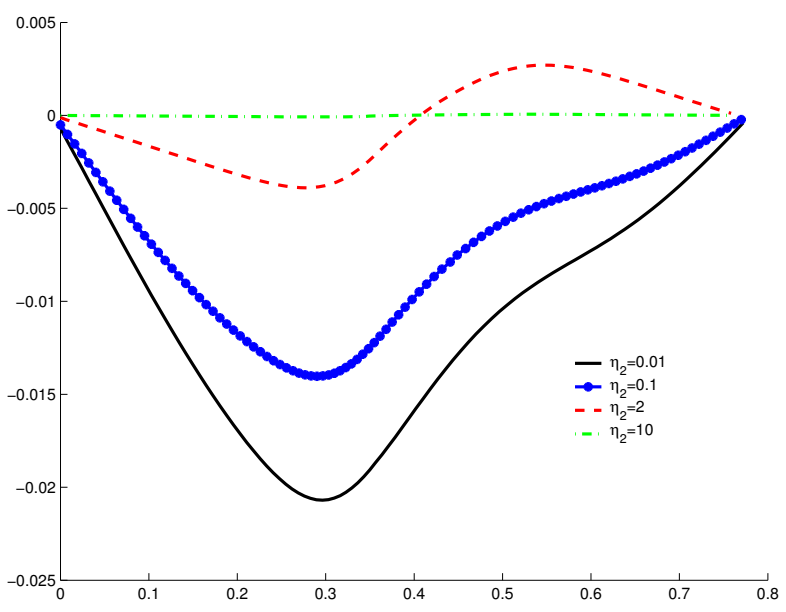

(b) $t=1 \mathrm{~s}$.

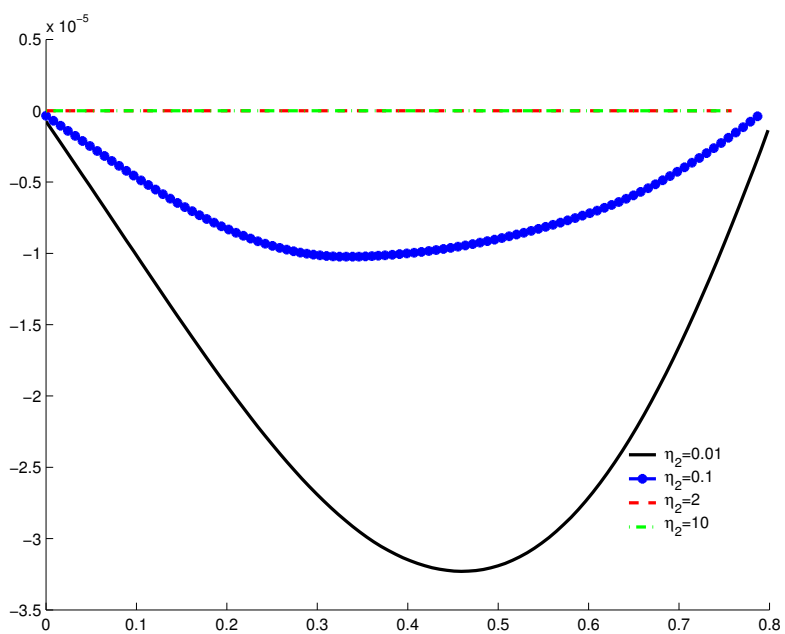

(d) $t=20 \mathrm{~s}$. (Range of the $y$-axis multiplied by $10^{-5}$ )

Figure 8: Test 1: Velocities obtained for $\tau_{y}=10,2,0.1$ and 0.01 


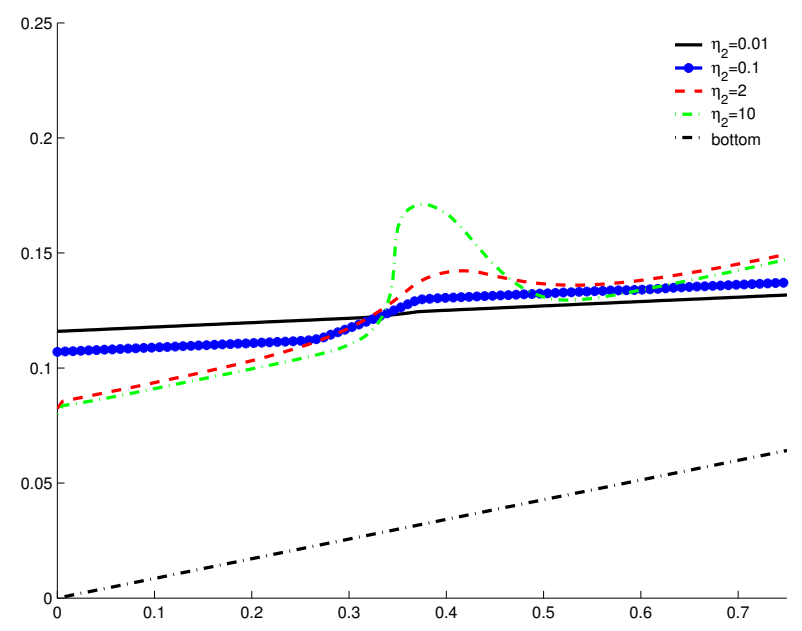

(a) Material surface.

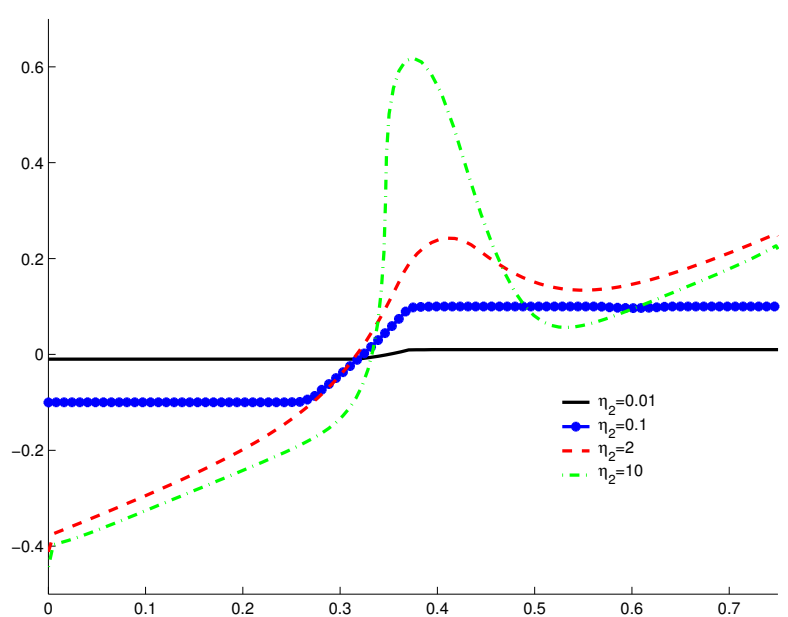

(b) $\mu$.

Figure 9: Test 1: Left: Material surface, Right: $\mu$, for the stationary solutions obtained with $\tau_{y}=10,2,0.1$ and 0.01

modify this material and introduce this type of change in its properties.

We consider a domain of 2 meters over an inclined slope of 20 degrees. First we compute numerically the stationary solution for $\tau_{y}=10$ when the initial condition is

$$
H(x, 0)= \begin{cases}2.1 & \text { if } x \in[1.5,1.6] \\ 0.1 & \text { otherwise }\end{cases}
$$

Then, we consider this stationary solution as initial condition for the same problem, except we change the value of $\tau_{y}$. Namely, we consider $\tau_{y}=5$ and $\tau_{y}=2$. In Figure 10, we see the transitions between the stationary solution (continuous black line, labelled "initial condition") when we change the rigid properties of the material. For $\tau_{y}=2$ (Figure 10(a)) we observe that the transition is from a bump to a stationary solution defined by two areas of horizontal free surface and an inclined surface connecting these areas. For $\tau_{y}=5$ (Figure 10(b)) the transition leads to a similar bump shape with only a change of the height and the width of the bump.

\subsection{Test 3: avalanche}

In this test, we consider a domain of 10 meters. The final time is $T=2 \mathrm{~s}$. The angle of the inclined plane that defines the bottom is 30 degrees, and $\tau_{y}=10$. As initial conditions we consider a velocity which is equal to zero in all the domain and $H$ defined by

$$
H(x, 0)= \begin{cases}10.1 & \text { if } x \geq 9.5 \\ 0.1 & \text { otherwise }\end{cases}
$$

In Figure 11, we present the evolution of the free surface for $t=0.3,0.5,1,1.5$ and 2 s. We observe that contrary to the two previous tests for the same value of $\tau_{y}$, in this case we do not obtain a stationary solution with a bump shape. There are several factors which induce the avalanche of the material in this test. First, the angle that defines the bottom is bigger in this case. Second, the height of the jump in $H$ at the initial condition. 


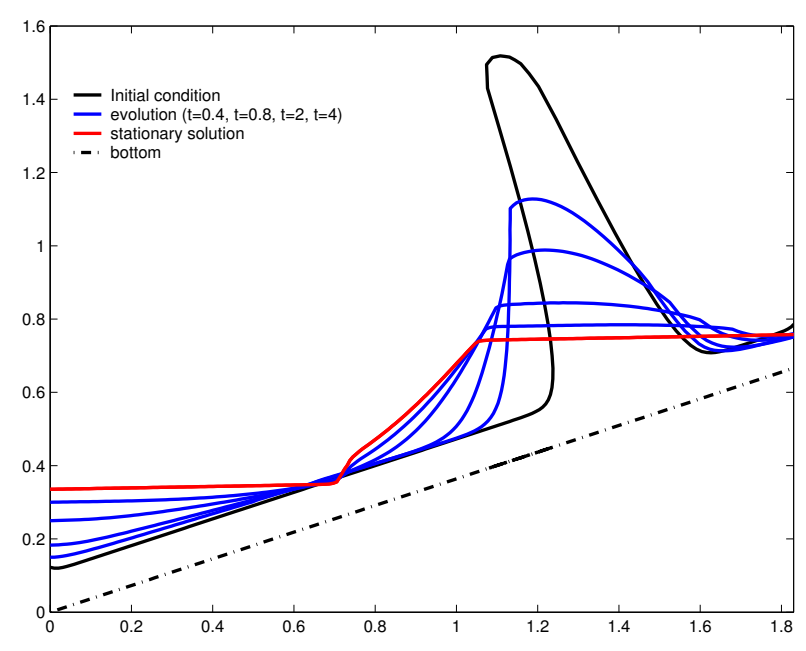

(a) $\tau_{y}=2, \theta=20^{\circ}$.

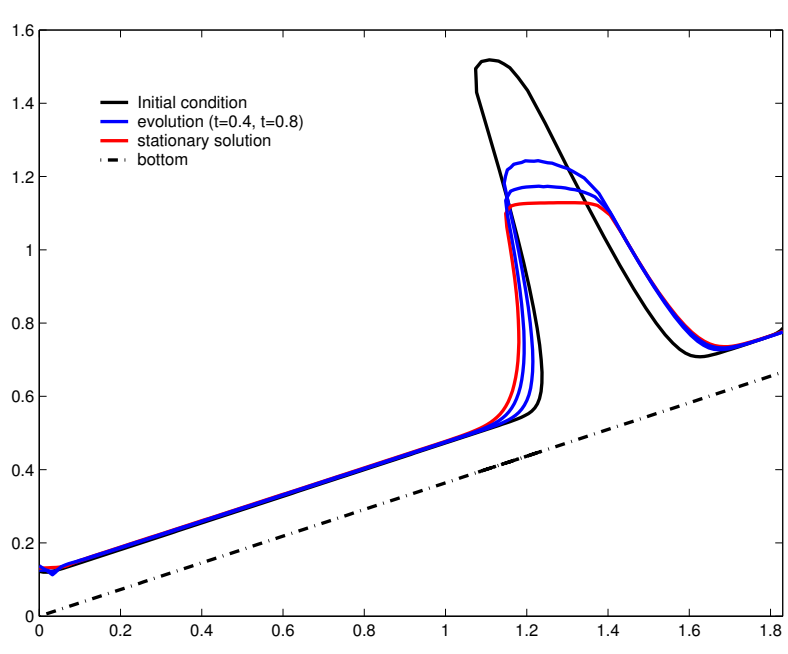

(b) $\tau_{y}=5, \theta=20^{\circ}$.

Figure 10: Test 2: Free surface. Transition between two stationary solutions. Continuous black line : first stationary solution (used as initial condition for the second run, see text). Blue lines: evolution of the free surface. Red line: second stationary solution.

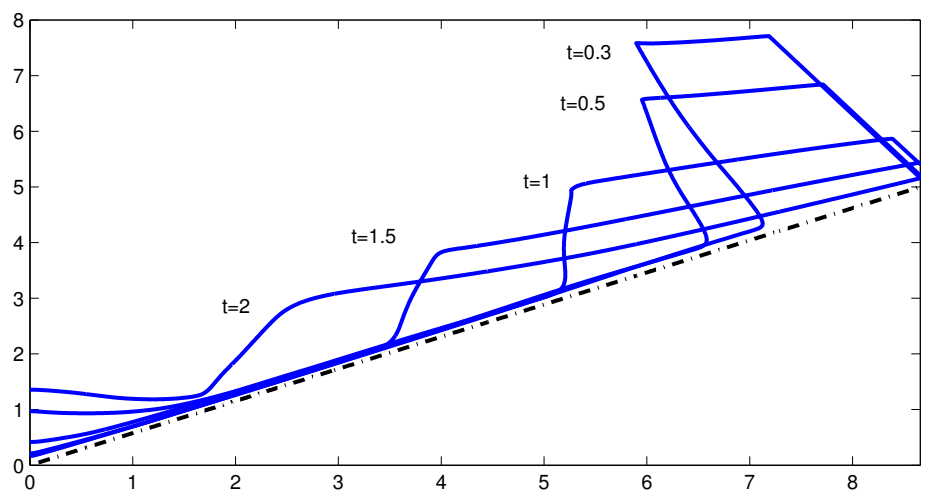

Figure 11: Test 3: avalanche. Surface evolution.

And finally, by condition (35) we observe that the length of the domain is also important. Even in the zone where the height of the material is constant, in $x \in[0,9.5)$, the solution is not stationary.

In Figure 12, we compare the surface at $t=1 \mathrm{~s}$. and $t=2 \mathrm{~s}$. with the value of the Lagrange multiplier $\mu$. To picture both quantities in the same range we have multiplied $\mu$ by 0.3 . We observe that the value of $\mu$ tends to follow the profile of the surface, in order to compensate the gradient of pressure. Nevertheless in this case, contrary to the Test 1 , it is not sufficient to block the flow and an avalanche of the material occurs all over the domain.

Acknowledgements. The first author would like to thank the Institut de la Montagne (Université de Savoie) managed from the research point of view by Carmen de Jong for financial support through the PPF Université de Savoie: "Mathématiques et avalanches de 


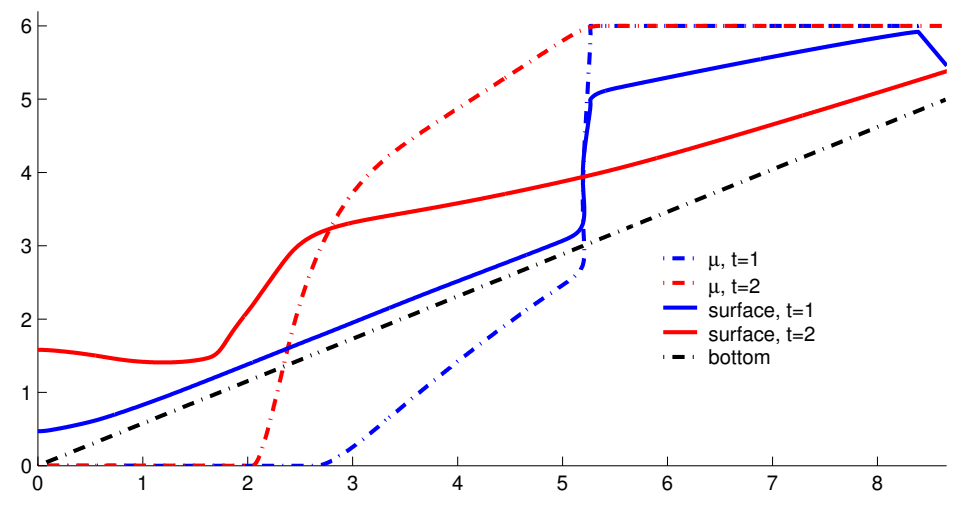

Figure 12: Test 3: Comparison of the free surface and the Lagrange multiplier

neige, une rencontre possible ?". He is also supported by the ANR project "MathOcean" 2008-2011. The second author has been partially supported by the Spanish Government Research project MTM2006-01275 and the Région Rhône-Alpes (France). The last author would like to thank the CNRS for the post-doctoral position he held during the academic year 2007/2008 when this study has been initiated.

\section{References}

[1] C. Ancey. Plasticity and geophysical flows: A review. Journal of Non-Newtonian Fluid Mechanics, 142:4-35, 2007. 3

[2] C. Ancey et al. Dynamique des avalanches. Presses Polytechniques et Universitaires Romandes - CEMAGREF, 2006. 2

[3] N. Balmforth, R. Craster, and R. Sassi. Shallow viscoplastic flow on an inclined plane. J. Fluid Mech., 470:1-29, 2002. 2

[4] N. Balmforth, R. Craster, and R. Sassi. Dynamics of cooling viscoplastic domes. J. Fluid Mech., 499:149-182, 2004. 2

[5] I. Basov and V. Shelukhin. Generalized solutions to the equations of compressible Bingham flows. Z. Angew. Math. Mech., 79(3):185-192, 1999. 3, 6

[6] I. V. Basov. Existence of a rigid core in the flow of a compressible Bingham fluid under the action of a homogeneous force. J. Math. Fluid Mech., 7(4):515-528, 2005. 3,6

[7] I. V. Basov. Long-time behavior of one-dimensional compressible Bingham flows. $Z$. Angew. Math. Phys., 57(1):59-75, 2006. 3, 6

[8] A. Bermúdez and M. E. Vázquez Cendón. Upwind methods for hyperbolic conservation laws with source terms. Comput. Fluids, 23(8):1049-1071, 1994. 13

[9] E. C. Bingham. Fluidity and Plasticity. Mc Graw-Hill, First edition, 1922. 3 
[10] M. Boutounet, L. Chupin, P. Noble, and J. Vila. Shallow water viscous flows for arbitrary topography. Communications in Mathematical Sciences, 6(1):29-55, March 2008. 3

[11] M. Castro-Díaz, T. Chacón-Rebollo, E. Fernández-Nieto, and C. Parés. On wellbalanced finite volume methods for nonconservative nonhomogeneous hyperbolic systems. SIAM J. Sci. Comput., 29(3):1093-1126, 2007. 13, 18, 19, 20

[12] O. Cazacu and N. Cristescu. Constitutive model and analysis of creep flow of natural slopes. Italian Geotechnical Journal, 34:44-54, 2000. 5

[13] O. Cazacu and I. R. Ionescu. Compressible rigid viscoplastic fluids. J. Mech. Phys. Solids, 54(8):1640-1667, 2006. 3

[14] O. Cazacu, I. R. Ionescu, and T. Perrot. Numerical modeling of projectile penetration into compressible rigid viscoplastic media. Int. J. Numer Meth. Engng., 74(8):1240 $-1261,2007.17$

[15] N. Cristescu. Plastic flow through conical converging dies, using a viscoplastic constitutive equation. Int. J. mech. Sci., 17:425-433, 1975. 5

[16] E. Dean, R. Glowinski, and G. Guidoboni. On the numerical simulation of Bingham visco-plastic flow: old and new results. Journal of Non Newtonian Fluid Mechanics, 142:36-62, 2007. 3

[17] G. Duvaut and J. Lions. Les inéquations en mécanique et en physique. Dunod, Paris, 1972. 6

[18] E. Fernández-Nieto, P. Noble, and J. Vila. Shallow water equations for nonNewtonian fluids. Submitted, 2008. 3

[19] M. Fortin and R. Glowinski. Méthodes de Lagrangien Augmenté - Applications à la résolution numérique de problèmes aux limites. Méthodes Mathématiques de l'Informatique. Dunod, 1982. 13, 14, 16

[20] J.-F. Gerbeau and B. Perthame. Derivation of viscous Saint-Venant system for laminar shallow water; numerical validation. Discrete Contin. Dyn. Syst., Ser. B, 1(1):89-102, 2001. 3, 10

[21] J. M. Greenberg and A.-Y. Le Roux. A well-balanced scheme for the numerical processing of source terms in hyperbolic equations. SIAM J. Numer. Anal., 33(1):116, 1996. 13

[22] R. J. LeVeque. Balancing source terms and flux gradients in high-resolution Godunov methods: the quasi-steady wave-propagation algorithm. J. Comput. Phys., 146(1):346-365, 1998. 13

[23] A. Mamontov. Existence of global solutions to multidimensional equations for Bingham fluids. Math. Notes, translation from Mat. Zametki 82, No. 4, 560-577 (2007), $82(4): 501-517,2007.3$ 
[24] C. C. Mei and M. Yuhi. Slow flow of a Bingham fluid in a shallow channel of finite width. J. Fluid Mech., 431:135-159, 2001. 2

[25] J. Oldroyd. A rational formulation of the equations of plastic flow for a Bingham solid. Proc. Camb. Philos. Soc., 43:100-105, 1947. 5

[26] S. P. Pudasaini and K. Hutter. Avalanche Dynamics - Dynamics of Rapid Flows of Dense Granular Avalanches. Springer, 2007. 2, 3

[27] P. L. Roe. Upwind differencing schemes for hyperbolic conservation laws with source terms. In Nonlinear hyperbolic problems (St. Etienne, 1986). C. Carraso et al. (Eds), volume 1270 of Lecture Notes in Math., pages 41-51. Springer, Berlin, 1987. 13

[28] V. V. Shelukhin. Bingham viscoplastic as a limit of non-Newtonian fluids. J. Math. Fluid Mech., 4(2):109-127, 2002. 3, 6

[29] J.-P. Vila. Sur la théorie et l'approximation numérique des problèmes hyperboliques non-linéaires, application aux équations de Saint-Venant et à la modélisation des avalanches denses. PhD thesis, Université Paris VI, 1986. 2

[30] G. Vinay, A. Wachs, and J.-F. Agassant. Numerical simulation of weakly compressible bingham flows: The restart of pipeline flows of waxy crude oils. Journal of Non-Newtonian Fluid Mechanics, 136(23):93 - 105, 2006. 13 